\title{
DIFERENCIAS INDIVIDUALES EN REGULACIÓN EMOCIONAL Y MENTALIZACIÓN EN PREADOLESCENTES CON TENDEN- CIAS A LA PSICOPATÍA
}

\author{
Ma José Paz Míguez \\ Clotilde SIneiro García \\ Facultad de Psicología. Universidad de Santiago de Compostela
}

\section{RESUMEN}

En una muestra de preadolescentes de 12 a 15 años analizamos si las relaciones de los rasgos conductual, afectivo e interpersonal de psicopatía -evaluados mediante el autoinforme "Youth Psychopathic Traits Inventory"- con los problemas atencionales $y$ las conductas agresiva y delictiva se ven alteradas -en intensidad $y / 0$ en dirección- en función del estatus emocional, mentalista y genérico del preadolescente vía labilidad / negatividad. Los resultados muestran cómo: a) la labilidad emocional y afectividad negativa actúa como un mecanismo mediador en la actuación del rasgo grandiosidad / manipulación sobre agresión, b) la interacción entre la alta competencia en regulación emocional y la alta competencia en inferencia de tristeza de segundo orden actúa como un proceso de riesgo en la actualización del rasgo grandiosidad / manipulación en chicas, c) la interacción entre la baja competencia en regulación emocional y la alta competencia en inferencia de

Correspondencia: Me José Paz Miguez. Facultad de Psicología. Universidad de Santiago de Compostela. 15782 Santiago de Compostela. Tel.: 981563100 Ext.: 13739.E-mail.: pc3739@usc.es. 
tristeza de segundo orden actúa como un proceso de riesgo en la actualización de los rasgos crueldad / insensibilidad y grandiosidad / manipulación en chicas, y d) la interacción entre la baja competencia autorreguladora y la alta competencia mentalista de tristeza actúa como un proceso de protección en la actuación del rasgo impulsividad / irresponsabilidad en chicas sobre problemas atencionales.

Palabras clave: RASGOS DE PSICOPATIA, GÉNERO, LABILIDAD/ NEGATIVIDAD, REGULACIÓN EMOCIONAL, TEORIA DE LA MENTE, PROBLEMAS DE CONDUCTA, PROBLEMAS DE ATENCIÓN.

\section{SUMMARY}

In a sample of boys and girls ages 12 to 15 years, we examined whether the relationship between psychopathic traits, self-reported in the "Youth Psychopathic Traits Inventory", and attention problems and extemalizing conduct undergoes changes, in strength or in direction, a function of the adolescent's emotional and mentalist status and generic status by way of lability / negativity. Regression analysis showed: a) the lability/negativity as mediator mechanism on the grandiosity / manipulation trait's action, b) the interaction between the high emotion regulation and the high sadnes's second order inference as a risk process on the grandiosity / manipulation trait's action in girls, c) the interaction between the low emotion regulation and the high sadnes's second order inference as a risk process on the callous / un emotional and grandiosity / manipulation traits's action in girls and d) the interaction between the low emotion regulation and the high sadnes's second order inference as a protective process on the impulsivity/irresponsibility trait's action in girls.

Key words: PSYCHOPATHY TRATTS, GENDER, LABILITY/EMOTIONALITY, EMOTIONAL REGULATION, THEORY OF MIND , CONDUCT PROBLEMS, ATTENTION PROBLEMS. 
La psicopatia, definida por características conductuales, interpersonales y afectivas, ha sido foco de interés en la psicología dada su relevancia clínica y social para la comprensión e intervención en la violencia y delincuencia severa adulta (Cleckley, 1941). A pesar de que la importancia de la identificación y prevención de niños y adolescentes en riesgo de desarrollar una personalidad y conducta psicopática fue resaltada ya por McCord y McCord (1959/1964) y la constatación de la severidad y la continuidad de los problemas de conducta de inicio temprano (Lahey y Loeber, 1994) e inicio demorado (Silverthorn y Frick, 1999), sólo recientemente se ha ampliado su estudio a la población infanto-juvenil. Su objetivo es favorecer el estudio sistemático de la etiología y precursores evolutivos de la psicopatía así como de los diversos factores de riesgo y protección que, operando mutua y transaccionalmente (Sameroff y Mackenzie, 2003), puedan explicar continuidades y discontinuidades y promover así el desarrollo de programas de prevención e intervención que los contemplen (Frick, 2001, 2004; Hinshaw, 2002; Salekin y Frick, 2005; Viding, 2004).

La aplicación adaptada a población infanto-juvenil de pruebas de evaluación de la psicopatía en adultos (Andershed, Kerr, Stattin y Levander, 2002; Frick, Bodin y Barry, 2000; Kosson, Cyterski, Steuerwald, Newman y Walter-Matthews, 2002) ha reflejado una estructura trifactorial. Además de identificar el factor "impulsividad/ irresponsabilidad", relacionado con el trastorno de conducta y trastorno de personalidad antisocial, se diferencian del factor afectivointerpersonal el de "crueldad / no emocionalidad" y el de "grandiosidad / manipulación", constituyentes de la esencia de la psicopatía (Cleckley, 1941). Estimadas individualmente como característica central de dicho concepto (Barry, Frick, DeShazo, McCoy, Ellis y Loney, 2000; Gustafson y Ritzer, 1995; Lynam, 1998), la consideración de las tres dimensiones parece inexcusable cuando de lo que se trata es de explicar la heterogeneidad psicológica y comportamental observada en los trastornos de conducta (Barry y cols., 2000; Barry, Frick y Killian, 2003; Blair, 1999; Frick, Cornell, Bodin, Dane, Barny y Loney, 2003; Frick, Stickle, Dandreaux, Farell y Kimonis, 2005; Lynam, 1998). Es interesante analizar, por to tanto, el papel potencial de estos factores en desadaptación comportamental en muestras normativas 
con objeto de identificar procesos y mecanismos que expliquen y modifiquen su capacidad operativa.

A este respecto, y en base al modelo teórico del Mecanismo de Inhibición a la Violencia (Blair, 1995; Blair y Frith, 2000) que considera el deterioro en empatía central en la psicopatía así como su asociación con las dimensiones afectiva (Pardini, Lochman y. Frick, 2003) e interpersonal de ésta (Millon, 1997), estudiamos dos de los procesos cuya normalidad implican empatía: teoría de la mente y regulación emocional (Decety y Jackson, 2004; Eisenberg, 2000). Ambos, el conocimiento de los estados emocionales y la regulación emocional podrían contribuir y potencialmente moderar el desarrollo de la responsividad empática (Denham, 1986) la cual a su vez, valorada como preocupación por los otros, juega un papel protector en la severidad de problemas externalizantes (Hastings, Zahn-Waxler, Robinson, Usher y Bridges, 2000). Asimismo, el desarrollo de la capacidad de mentalización está vinculada a las funciones ejecutivas - a la autorregulación afectiva (Russell, 1996). En esta medida, nuestra propuesta es que ambos procesos podrían estar comprometidos individual $e$ interdependientemente en las diferentes trayectorias que conducen desde la tendencia psicopática a la heterogeneidad conductual en adolescentes de ambos géneros, abarcando ésta desde sintomatología externalizante a normalidad.

\section{PSICOPATÍA Y REGULACIÓN EMOCIONAL}

La regulación emocional, organizadora del funcionamiento psicológico y definida por procesos internos y externos implicados en iniciar, motivar, mantener y modular la experiencia y expresión emocional de manera socialmente adaptativa (Campos, Frankel y Camras, 2004; Eisenberg y Morris, 2002; Thompson, 1994), constituye una tarea evolutiva esencial en el desarrollo socioemocional y conductual (Cicchetti, Ackerman y (zard, 1995). El papel predictor de la labilidad y negatividad, particularmente la expresión de alto nivel de cólera, irritabilidad y tristeza, así como de su control voluntario inhibitorio deficitario y de la impulsividad en sintomatología externalizante se ha constatado en una importante serie de investigaciones (Eisenberg, Cumberland, Spinrad, Fabes, Shepard, Reisser, Murphy, Losoya, Guthrie, 2001; Eisenberg, 
Sadovsky, Spinrad, Fabes, Losoya, Valiente, Reiser, Cumberland y Shepard, 2005; Lengua, 2003; Shields y Cicchetti, 1998).

Los estudios con niños y jóvenes con problemas de conducta y alta impulsividad / irresponsabilidad señalan la presencia de problemas en regulación emocional (Frick y Morris, 2004) expresados en reactividad emocional elevada y afectividad negativa -lo que sugiere un patrón de infrarregulación emocional (Cole, Michel y Teti, 1994)(Frick, Lilienfeld, Ellis, Loney y Silverthorn, 1999; Loney, Frick, Clements, Ellis y Kerlin, 2003), desregulación conductual (Pardini y cols., 2003) y agresión reactiva (Frick y Ellis, 1999). En la medida en que este patrón de desregulación emocional e impulsividad explica una de las trayectorias evolutivas a los problemas de conducta severos (Caspi, Moffitt, Newman y Silva, 1998; Frick y Morris, 2004) y a una personalidad resistente (Eisenberg y cols., 2004), consideramos relevante determinar el papel específico que juegan los componentes temperamental y socializado de regulación emocional en el desarrollo de los problemas de conducta.

Asimismo, y aun cuando la investigación del rasgo narcisista como factor de riesgo de desadaptación en población infanto-juvenil está en sus comienzos (Barry, Frick y Killiam, 2003), el perfil temperamental del narcisista caracterizado por baja tolerancia a la frustracción, labilidad y reactividad emocional alta ante indicios sociales que desafían su autoestima elevada pero inestable y frágil (Rhodewalt y Morf, 1998) parece sugerir un patrón de infraregulación emocional. Complementariamente, y en la medida en que los síntomas son reflejo de experiencia emocional y conductas que la manejan (Bradley, 2000), los comportamientos narcisistas al servicio de la autorregulación y mantenimiento de la autoestima (Morf y Rhodewalt, 2001) podrían ser considerados como estrategias de regulación emocional inadecuadas que proporcionan alivio inmediato del malestar atenuando el impacto afectivo de tales indicios pero manteniendo y/o agravando disfunciones intra e interpersonales (Strauman, 2001).

No obstante, la literatura dista de ser consistente respecto al perfil temperamental y de regulación emocional en población infanto-juvenil con problemas de conducta. Los estudios de niños y jóvenes antisociales con rasgo crueldad / insensibilidad muestran una inhibición conductual baja (Barry y cols., 2000; Frick y cols., 2003), que 
cumple un papel fundamental en el deterioro de la empatía (Kochanska, 1997) y en el desarrollo de la psicopatia (Blair, 1999). En este sentido, trabajos de Eisenberg y cols. (ver Eisenberg, 2000) indican cómo la experiencia de tristeza y los procesos regulatorios voluntarios se relacionan positivamente con la empatía y cómo la experiencia de miedo y ansiedad pueden ser factores protectores de conducta criminal.

Los niños con problemas de conducta y alto nivel de crueldad / insensibilidad muestran reducida afectividad negativa (Frick y cols., 1999), reducida responsividad psicofisiológica a expresiones faciales de tristeza (Blair, 1999) y débil reactividad a estimulación emocional o eventos negativos (Barry y cols., 2000; Loney y cols., 2003) así como dificultad en el reconocimiento y sensibilidad a expresiones de miedo y tristeza (Blair, Colledge, Murray y Mitchell, 2001). El reducido nivel de arousal puede reflejar un problema en regulación emocional dado la aparente incapacidad para acceder a emociones específicas en situaciones aversivas -dimensión de desregulación (Cole, Michel y Teti, 1994)- y la insuficiente modulación afectiva -inhabilidad para activar y/o intensificar el estado y expresión emocional (Cole y cols., 1994), lo que podría indicar un patrón de sobrerregulación emocional. En consecuencia, es posible que diferentes e incluso opuestos patrones de reactividad emocional (Loney y cols., 2003) y de desregulación emocional puedan estar implicados en cada una de las vías a los problemas de conducta de inicio temprano. Además, y consistente con el deterioro de componentes básicos de la empatía afectiva -responsividad emocional y conocimiento emocional (Feshbach, 1987)-, la preocupación empática y angustia por los efectos de su conducta en otros predicen inversamente la presencia de rasgos afectivos en jóvenes antisociales (Pardini y cols., 2003).

La literatura de la competencia emocional en el "bullying", en el que es manifiesto un patrón de deterioros emocionales similar al observado en los niños con tendencia psicopática, sugiere problemas en regulación emocional (Shields y Cicchetti, 2001), particularmente, un patrón de sobrerregulación emocional. 


\section{PSICOPATÍA Y TEORÍA DE LA MENTE}

La habilidad para comprender estados informacionales y emocionales ajenos constituye otra de las tareas evolutivas esenciales (Riviére y Núñez, 1996). No obstante, la investigación de su relación con los problemas de conducta infanto-juveniles es escasa y, todavía más, con la psicopatía. Los resultados indican que la adquisición mentalista tiene implicaciones tanto positivas como negativas de distinta naturaleza en competencia social (Cutting y Dunn, 2002; Fahie y Symons, 2003; Sutton, Smith y Swettenham, 1999) lo que plantea una relación compleja (Astington, 2003). Se ha observado algún índice de deterioro mentalista (Happé y Frith, 1996; Hughes, Dunn y White, 1998) así como un sesgo en lectura mental en niños y preadolescentes con conducta disruptiva. El sesgo "teoría de las mentes malignas" se infiere de la mayor capacidad mentalista en conducta antisocial que social adaptativa (Happé y Frith, 1996), de la mayor inferencia emocional bajo contexto de sorpresa desagradable que agradable (Hughes y cols., 1998) y de la relación positiva de la teoría de la mente con negación de responsabilidad y ausencia de remordimiento (Sutton, Reeves y Keogh, 2000). Además, este perfil mentalista, coherente con conductas como la mentira, la manipulación de los otros o la culpa ajena que requieren conocimiento y manipulación de las mentes, concuerda con el planteamiento de Repacholi y Slaughter (2003) y Sutton y cols. (1999). Éstos plantean que muchos "bullies" pueden ser buenos lectores de mente ante objetivo antisocial dada su superioridad en competencia mentalista de 2 orden y su tendencia a la agresión proactiva e indirecta. A pesar de ello, el estudio de jóvenes antisociales con tendencia a la psicopatía muestra cómo el rasgo crueldad / insensibilidad se relaciona negativamente con la toma de perspectiva además de con la preocupación empática (Pardini y cols., 2003). No obstante, hay que tener en cuenta en la valoración de este resultado que, como en la mayoría de trabajos en esta área, no se ha considerado la contribución individual del rasgo grandiosidad I manipulación, ni se han aislado el efecto de cada dimensión de psicopatía respecto al de los problemas de conducta asociados, ni el efecto aditivo o interactivo de procesos psicológicos normativamente relacionados. 
Por lo tanto, y sin desestimar la presencia de deterioro mentalista en niños con problemas de conducta, debemos considerar la relevancia que potencialmente adquiere el incremento de mentalización en la conducta antisocial bajo condición de ausencia de disposición empática (Hoffman, 1975). Así, si la ausencia de empatía es central en psicopatia y a su vez se identifica como factor general de empatía la reactividad emocional empática (Cliffordson, 2002), deteriorada en jóvenes crueles (Pardini y cols., 2003), es la baja responsividad emocional empática más que el conocimiento mentalista de los niños la que podría actuar como mediador y éste, como posible amplificador del efecto de los rasgos afectivo e interpersonal de psicopatía en el contexto de desrregulación emocional.

Paralelamente, los estudios con niños con TDAH informan tanto de ausencia de deterioro mentalista (Perner, Kain y Barchfeld, 2002) como de dificultades en inferencia cognitiva y emocional de $2^{\circ}$ orden (Buitelaar, Van Der Wess, Swaab-Barneveld y Jan Van Der Gaga, 1999). Se observó asimismo la relación positiva del funcionamiento ejecutivo con teoría de la mente (Fahie y Symons, 2003; Perner y cols., 2002), planteándose que puedan suceder asociaciones directas $e$ indirectas entre mal funcionamiento ejecutivo y conducta disruptiva (Hughes, Dunn y White, 1998). No obstante, no sólo debemos considerar el efecto combinatorio de estas competencias sino también, y especialmente, su efecto condicional en síntomas, pudiendo pensar en teoria de la mente como un posible moderador o amortiguador del efecto del rasgo conductual de psicopatía dependiente del nivel de desregulación emocional.

Los datos, todavía insuficientes, sobre la naturaleza de la relación teoría de la mente / relaciones sociales concitan el interés sobre el estudio de la conducta antisocial y psicopatía si de lo que se trata es que precisar la naturaleza y alcance de esa relación en la explicación de vías alteradas de funcionamiento. Por lo tanto, se deben explicar los nexos de teoría de la mente con competencia social y funcionamiento ejecutivo $y$, al mismo tiempo, con desadaptación externalizante. La propia complejidad del desarrollo requiere su explicación en el marco en el que se permita valorar el impacto no solo acumulativo sino también interactivo de diversos sistemas humanos básicos como factores de riesgo y protección a lo largo del curso 
evolutivo (Masten, 2001). El esclarecimiento de la relación entre estos procesos, en términos de diferencias individuales, constituye el objetivo general de nuestro estudio. De acuerdo con ello, y en base a la revisión comentada, planteamos que:

1. La elevada labilidad y negatividad en las reacciones emocionales de los jóvenes podría ser la base sobre la que se construyen los rasgos de impulsividad / irresponsabididad y de grandiosidad / manipulación que les hacen proclives a problemas de conducta y, de igual manera, su baja labilidad y negatividad es la que lo podría explicar respecto al rasgo crueldad / no emocionalidad. Esto es, el componente temperamental de regulación emocional podría ser uno de los mecanismos mediadores que explican el efecto de los rasgos psicopáticos.

2. La interdependencia regulación emocional y teoría de la mente podrían modificar o moderar la intensidad y dirección del efecto de cada uno de los rasgos de psicopatía en desadaptación conductual. Específicamente:

2.a. Regulación emocional podría ser uno de los procesos que ejercerían un papel modulador o moderador de los sistemas más reactivos si tomamos en consideración las propuestas de Rothibart y Bates (1998) así como los resultados de los trabajos de Eisenberg y cols. (ver Eisenberg, Fabes, Guthrie y Reiser, 2002) y de Blair, Denham, Kochanoff y Wipple (2004). Cabría pensar que una regulación emocional sensible y responsiva a las demandas contextuales pueda ser capaz de mitigar o amortiguar el efecto que los distintos rasgos psicopáticos ejercen en problemas de conducta dada la responsividad emocional disposicional elevada y reducida y los consiguientes patrones de infra y sobre regulación emocional implicados en su actualización.

2.b. Teoria de la mente, evaluada en su multicomponencialidad bajo contexto de "sorpresa desagradable", podría ser uno de los mecanismos responsables del impacto diferencial de cada uno de los rasgos de psicopatía en problemas de conducta. Particularmente, si tenemos en mente el modelo de Blair (1995) respecto al procesamiento emocional diferencial cabría pensar que el componente de inferencia de tristeza en el otro intensificaría la magnitud del efecto de los rasgos crueldad / insensibilidad y grandiosidad / manipulación en desadaptación, mientras que el componente de inferencia de 
creencia lo haría para este último rasgo dado el requerimiento mentalista de las conductas implicadas en el mismo. Podríamos deducir asimismo que la inferencia de tristeza podría mitigar o amortiguar el efecto del rasgo conductual en desadaptación. No obstante, tanto los efectos amplificadores como los amortiguadores ocurrirían bajo las condiciones naturales de dichos rasgos -en ausencia de reactividad y regulación emocional adecuadas-. En los casos de competencia en regulación emocional en presencia de reactividad y regulación emocional adecuadas-, los efectos amplificadores de la mentalización podrían reducirse e invertirse mientras que los efectos amortiguadores, intensificarse.

2.c. Cualquier efecto de moderación que la teoría de la mente en interdependencia con la auto-regulación emocional ejerza sobre la capacidad operativa de los rasgos psicopáticos sucedería, al menos parcialmente, a través del efecto mediador de la labilidad /afectividad negativa.

3. Las diferencias genéricas en prevalencia e inicio de los problemas de conducta, y en curso, severidad y factores de riesgo asociados diferencialmente a las dos trayectorias evolutivas tradicionales, asl como los modelos evolutivos que las contemplan (Moffitt y Caspi, 2001; Silverthorn y Frick, 1999) sugieren que el género podría ser un moderador de la influencia de los rasgos psicopáticos en desadaptación externalizante. Asimismo, y dado que "no está claro si o no el peso e interacción de los factores de riesgo operan similarmente para chicos que para chicas" (Odgers y Moretti, 2002, pp.112), examinanos los efectos interactivos potenciales de riesgo y protección dependientes o específicos de género.

\section{MÉTODO}

\section{Muestra}

La muestra está compuesta por 233 adolescentes de entre 12 y 15 años (media = 12:6 años) pertenecientes a diferentes CEIPS e IES de Pontevedra y Santiago. Respecto al género, el $55.4 \%$ de la muestra son niños y el $43.8 \%$, niñas. Como criterios de selección se utilizaron edad y desarrollo normativo, estableciendo la edad mínima 
en 12 años por razón de la aplicación de un autoinforme del comportamiento psicopático.

El contacto con los centros se realizó mediante una entrevista con el director y el orientador en la que se presentaron los objetivos $\theta$ instrumentos de evaluación a realizar por tutor y adolescente y en la que se requirió su colaboración. Mediante una carta, enviada a través del centro, se contactó con los padres en la que además de solicitar su autorización se les explican los motivos de la evaluación. Obtenido el consentimiento, se procedió a la entrega de dos cuestionarios al tutor y a la aplicación en grupo de dos tareas de evaluación, registrando aula y número de identificación del alumno así como edad y género.

\section{Instrumentos de evaluación}

\section{Rasgos de psicopatía}

Para su evaluación se empleó una versión adaptada al español del "Youth Psychopathic Traits Inventory" (YPI, Andershed, Kerr, Stattin, Levanter, 2002). El YPI es un autoinforme de 50 items diseñado para medir los rasgos centrales de la psicopatía y en el cual se estima el grado de adecuabilidad de cada comportamiento en una escala Likert (de 1 "no es nada adecuada" a 4, "es muy adecuada"). Los items conforman 3 factores que valoran: a) atractivo superficial, grandiosidad, mentira y manipulación -"Grandiosidad/Manipulación" (Grand/Manip)-, b) no emocionalidad, ausencia de remordimiento y crueldad "Crueldad/No emocionalidad" (Cruel/NoEm)- y c) impulsividad, búsqueda de emociones e irresponsabilidad - "Impulsividad/ Irresponsabilidad" (Imp/lrrp). La consistencia interna para las escalas es alta $(\alpha=0.66-0.93)$ (Andershed y cols., 2002). Altas puntuaciones directas en una escala o dimensión indican presencia del rasgo correspondiente.

\section{Regulación emocional}

El "Emotion Regulation Checklist" (ERC, Shields y Cicchetti, 1997) valora autorregulación emocional en términos de responsividad y flexibilidad emocional y capacidad para modular la experiencia y 
expresión emocional adecuadamente en el contexto social. El tutor estima la frecuencia de comportamientos y estados emocionales del chico en una escala Likert ( 1 "nunca ocurre" a 4 "ocurre casi siempre"). Los 24 items, de los cuales retiramos el 12 y 20 dado su contenido conductual (Shields y Cicchetti, 1998; Comunicación personal, ) conforman 2 factores que captan variabilidad, inflexibilidad emocional y emociones desreguladas -"Labilidad / Negatividad" (Lab/Neg)- y expresión emocional adecuada, conocimiento emocional y empatía "Regulación emocional" (RegEm)-. La consistencia intema para ambas subescalas es alta ( $\alpha=.96$ y $\alpha=.83$ ) (Shields y Cicchetti, 1997). Para que altas puntuaciones indiquen niveles superiores de autorregulación, a todos los items con peso negativo se les invirtió la puntuación.

\section{Teoria de la mente: Inferencia de creencia falsa de segundo orden}

La tarea de falsa creencia de segundo orden de "la historia del heladero" (Perner y Wimmer, 1985) valora la habilidad para comprender que una persona sostiene un pensamiento, correcto o no, acerca de lo que piensa otra acerca de algo. La tarea implica la presentación escrita de una historia en la que mientras un niño va a casa a coger dinero para comprarle un helado al heladero del parque, su amiga observa cómo éste se va a la iglesia. En tanto la amiga está en el parque, el niño encuentra al heladero que le indica el cambio de lugar. A continuación, se plantean dos preguntas control -recuerdo y conocimiento de la realidad-, una pregunta de predicción de conducta y justificación mentalista de $2^{\mathbf{2}}$ orden - ¿ ¿Dónde piensa María que fue Juan a comprar el helado?", "¿Por qué?"- y otra de inferencia emocional y justificación mentalista de $2^{\mathbf{q}}$ orden - “ ¿Cómo piensa María que se siente Juan?", "¿Por qué?"-. Esta tarea puede ser categorizada como situación de sorpresa desagradable.

Las categorías son la de "Atribución de creencia" (Atr.Cre), "Inferencia de enfado" (Inf.Enf) e "Inferencia de tristeza" (Inf.Tris), valoradas sólo si las cuestiones control son correctas. En la primera, se asignó 0 ante respuesta ausente 0 incorrecta, 1 ante respuesta correcta -"parque"- y 2 si además se explica la predicción de conducta en términos de inferencia mentalista de $2^{2}$ orden, informando 
explícita o implicitamente de las cogniciones de ambos niños. En las categorías de inferencia emocional, se asignó 0 ante respuesta ausente o incorrecta, 1 ante respuesta correcta -"enfado"/4tristeza"- y 2 si además se justifica la inferencia emocional en términos de creencia de $2^{\circ}$ orden, informando de las cogniciones de ambos 0 , condicional a la anterior categoría, de la cognición de la niña y conducta del niño.

\section{Competencia conductual}

La evaluamos mediante las subescalas de problemas de atención, conducta agresiva $(\alpha=.94)$ y delictiva $(\alpha=.64)$ del "Teacher's Report Form" (TRF, Achenbach, 1991) dada su asociación con los rasgos psicopáticos. De cara a los análisis, se retiraron los items $77,86,87$ y 95 de la escala agresiva y el 45 de la de atención dado su contenido emocional y superposición artificial con los del ERC.

\section{Procedimiento estadístico}

Para determinar el papel moderador o mediador que los procesos de regulación emocional y mentalización pudieran jugar en el desarrollo de la psicopatía se valoró, en $1^{2}$ lugar, la intensidad y naturaleza de las relaciones entre las variables. Se realizaron, a continuación, una serie de análisis de regresión múltiple para determinar la contribución predictiva de los rasgos de psicopatía en desadaptación y el grado en que la regulación emocional, la teoria de la mente y el género alteran la dirección y/o intensidad del efecto del predictor moderación- y/o la labilidad/negatividad explica cómo se produce el efecto predictor -mediación- 0 el efecto moderador -moderación mediada- (Baron y Kenny, 1986). Para examinar el efecto de moderación se estimó un modelo de regresión por el método introducir para los efectos simples e interactivos del predictor (VI) y de las variables moderadoras (VMo) sobre cada una de las variable criterio (VD) resultando en tres modelos de regresión múltiple- Para ello, se dicotomizaron la dimensión de RegEm y las tres de ToM en base a las medias de las puntuaciones centradas -para evitar la multicolienalidad (Aiken y West, 1991)- (Whisman y McClelland, 2005) indicando 1 alta competencia y 0 baja competencia. Respecto al 
género, se asignó 0 al masculino y 1 al femenino. En cada modelo se introdujeron las $3 \mathrm{Vl}$, las $4 \mathrm{VMo}$, la Lab/Neg y las 2 variables demográficas continuas asi como los productos por orden de 2, 3 y 4 términos de las VI, VMo y género. La interpretación de estos efectos se basa en el coeficiente de regresión no estandarizado (B). La significación del B de la VI señala efecto simple condicional a las VMo del modelo fijadas en 0 , y la del $B$ de la interacción, efecto interactivo-debilitada o fortalecida-, indicando que el efecto del predictor sobre el criterio depende de los niveles de las VMo cuando la variable excluida en interacción es fijada en 0 (Aiken y West, 1991; Whisman y McClelland, 2005). En la interpretación de las interacciones de 2 y 3 vías significativas, además de la representación gráfica, se realizaron análisis adicionales para conocer la significación estadística de las pendientes simples asi como de sus diferencias (Aiken y West, 1991; Dawson y Richter, 2006).

Para examinar el efecto de mediación se estimó un modelo de regresión por introducir requiriendo de la significación de los coeficientes de regresión estandarizados (b) que refieren al: (a) efecto de la VI en la variable mediadora (VMe); (b) efecto de la VI en la VD, y (c) efecto de la VI en criterio reducido o anulado al introducir el efecto de VMe. Se examinó el efecto de moderación mediada mediante 3 modelos de regresión requiriendo la significación del: (a) efecto de la VMo; (b) efecto de la VMo sobre VMe y/o (c) efecto de la VMe moderado en VD y (d) efecto residual de la VMo en VD reducido/anulado.

\section{RESULTADOS}

\section{Intercorrelaciones}

En la tabla 1 se muestran las correlaciones entre todas las variables, sus medias y desviaciones típicas asi como las correlaciones parciales por edad.

Respecto a las correlaciones parciales, las dimensiones de psicopatía se relacionan positivamente entre sí y con algunas VD, indicando que a mayor nivel de Grand/Manip, mayor nivel de P.atención, C.delictiva y C.agresiva, que a mayor nivel de Cruel/NoEm, mayor nivel de P.atención y que a mayor nivel de $\mathrm{Imp} / \mathrm{rrp}$, mayor nivel de P.atención, 


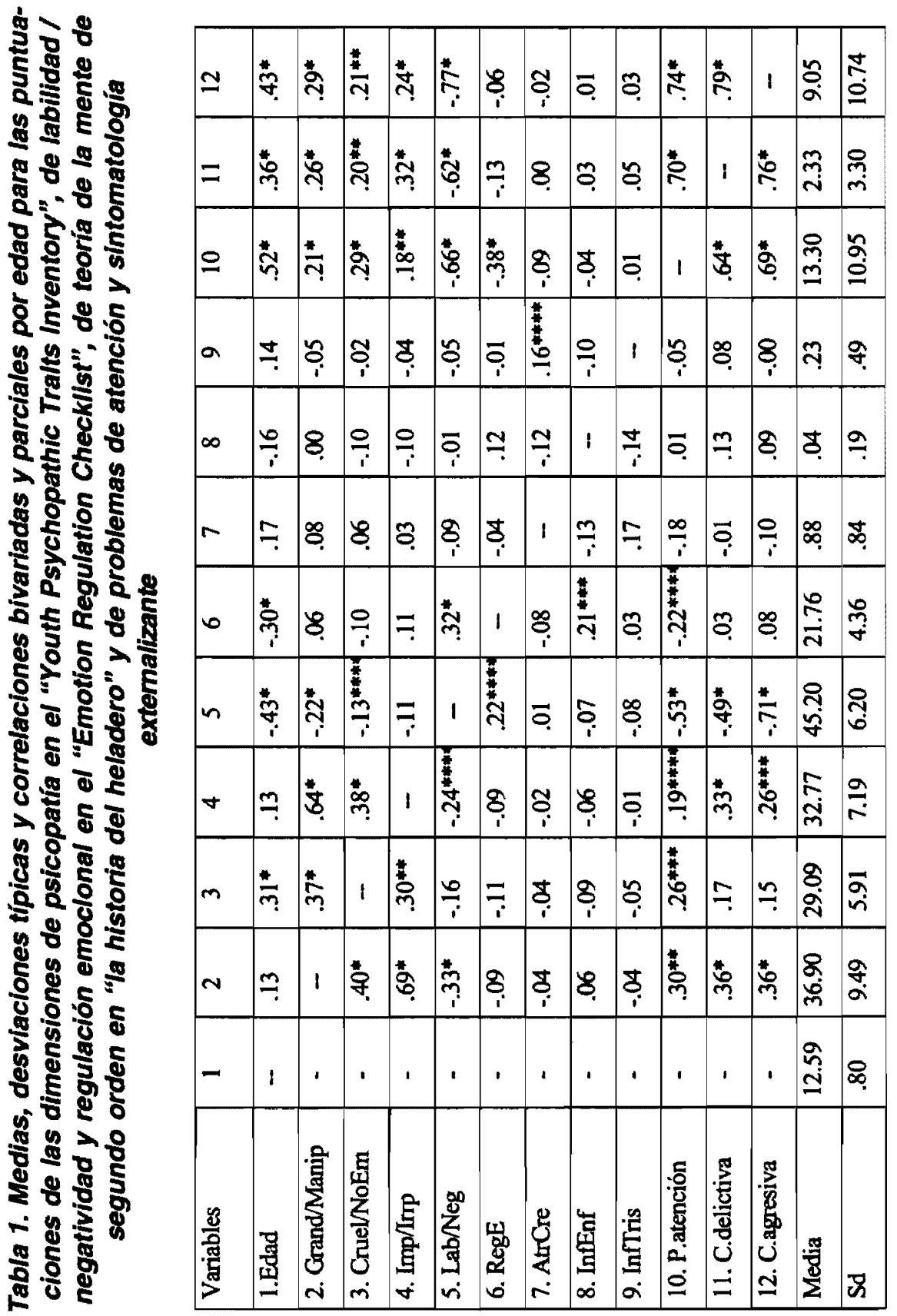


NOTA A LA TABLA 1: En la tabla Grand/Manip = grandiosidad / manipulación; Cruel/NoEm = crueldad $/$ no emocionalidad; $/ \mathrm{mp} / \mathrm{lrrp}=\mathrm{impulsividad} /$ irresponsabilidad; Lab/Neg = labilidad / negatividad; RegEm = regulación emocional; AtrCre = atribución de creencia; InfEnf = inferencia de enfado; InfTris = inferencia de tristeza; $P$.atención = problemas de atención; C.delictiva= conducta delictiva; C.agresiva = conducta agresiva; Sd.= desviación típica.

Las correlaciones bivariadas se sitúan en la parte superior de la diagonal mientras que las correlaciones parciales por edad -0 al controlar el efecto de la edad- se sitúan en la parte inferior de la diagonal.

En la columna 1 no aparecen coeficientes de correlación porque la variable edad se ha introducido como variable control en las correlaciones parciales. Nivel de significación: " $p<.001$ (bilateral), " $p<.005$ (bilateral), "**" $p<.01$ (bilateral), ${ }^{* * * *} p<.05$ (bilateral)

C.delictiva y C.agresiva. La dimensión psicopática menos relacionada con desadaptación externalizante es la de Cruel/NoEm. Por otro lado, los rasgos de Grand/Manip e Imp//rrp se relacionan negativamente con $\mathrm{Lab} / \mathrm{Neg}$, indicando que jóvenes con mayores tendencias a la grandiosidad/manipulación asi como a la impulsividad/irresponsabilidad en su conducta tienen puntuaciones inferiores en la escala de Lab/ Neg -mayores niveles de labilidad/afectividad negativa-. A su vez, la Lab/Neg se asocia con P.atención, C.delictiva y C.agresiva. Respecto a las relaciones parciales de psicopatía y RegEm y de ésta con las VD sólo alcanza significación la relación RegEm con P.Atención, tal que elevada regulación emocional se asocia a reducida sintomatología atencional. Asimismo, ninguna de las tres categorias de ToM mantiene una relación significativa con psicopatía, Lab/Neg o desadaptación.

\section{Efectos simples y de moderación}

Las interacciones significativas de las dimensiones de RegEm, Inf.Trist y género con los rasgos de psicopatía explican todos los subtipos de problemas.

Específicamente, y respecto al modelo 1 , los efectos directos de Lab/Neg $(B=-1.069)$ y AtrCre $(B=-4.185)$ explicaron una cantidad significativa de la varianza de P.Atención, informando de un $33.9 \%$ y $8.7 \%$, respectivamente. Estas estimaciones indican que, independientemente del nivel de rasgo de psicopatía, baja Lab/Neg y Atr.Cre de 
Tabla 2. Resultados de los análisis de regresión múltiple por el método introducir de los ofectos simples e interactivos significativos de los rasgos de psicopatía, regulación emocional y teoría de la mente en problemas en atención. Modelo 1

\begin{tabular}{|c|c|c|c|c|c|c|}
\hline \multicolumn{7}{|c|}{ Problemas en Atención } \\
\hline \multirow[t]{2}{*}{ Modelo 1} & \multicolumn{2}{|c|}{$R 2=.776$} & \multicolumn{2}{|c|}{$F(55,50)=3.144$} & \multicolumn{2}{|c|}{ Sig. $=.000$} \\
\hline & $\mathbf{B}$ & SE B & $B$ & $\mathrm{t}$ & Sig. & R2parcial \\
\hline \multicolumn{7}{|l|}{ Variables } \\
\hline $\mathrm{Lab} / \mathrm{Neg}$ & -1.069 & .211 & -.520 & -5.076 & .020 & .339 \\
\hline AtrCre & -4.185 & 1.919 & -.301 & -2.180 & .034 & .087 \\
\hline $\begin{array}{l}\text { Cruel/NoEmx } \\
\text { Inf.Tristxgénero }\end{array}$ & 8.412 & 2.836 & 1.593 & 2.966 & .005 & .149 \\
\hline $\begin{array}{l}\text { lmp/Irrpx } \\
\text { Inf.Trisxgénero }\end{array}$ & -4.218 & 2.092 & -.985 & -2.016 & .049 & .075 \\
\hline $\begin{array}{l}\text { Cruel/NoEmx } \\
\text { RegEmx } \\
\text { Inf.Trisxgénero }\end{array}$ & -8.570 & 3.335 & -1.341 & -2.569 & .013 & .116 \\
\hline
\end{tabular}

NOTA: Para simplificar la presentación, en la tabla se presentan únicamente los efectos simples e interactivos significativos a $N=106$.

En la tabla Lab/Neg = labilidad / negatividad; AtrCre = atribución de creencia falsa de segundo orden; Cruel/NoEmxInf.Tristxgénero = interacción entre el rasgo crueldad / no emocionalidad, la competencia en inferencia de tristeza de segundo orden y el género; $/ \mathrm{mp} / \mathrm{l}$ rrpxinf. Trisxgénero = interacción entre el rasgo impulsividad / irresponsabitidad, la competencia en inferencia de tristeza de segundo orden y el género; Cruel/NoEmxflegEmxInf.Trisxgénero = interacción entre el rasgo crueldad / no emocionalidad, las competencias en regulación emocional y en inferencia de tristeza y el género.

$2^{9}$ orden predicen un menor nivel de P. atencionales. Además, el modelo 1 estimó un efecto significativo de la interacción Inf. Tris, género e Imp/irrp en P. Atención ( $B=-4.218$ ) -representada en la figura 1-, efecto que explica hasta un $7.5 \%$ de la varianza del criterio. Como muestran las pendientes simples significativas y las diferencias entre éstas, la relación positiva entre rasgo Imp/trp y P.Atención se hace mínima e incluso invierte cuando las chicas poco competentes 
Tabla 3. Resultados de los análisis de regreslón múltiple por el método introducir de los efectos simples e interactivos s/gniflcatlvos de los rasgos de psicopatía, regulación emoclonal y teoría de la mente en conducta delictiva. Modelo 2

\begin{tabular}{|c|c|c|c|c|c|c|}
\hline \multicolumn{7}{|c|}{ Conducta Delictiva } \\
\hline \multirow[t]{2}{*}{ Modelo 2} & \multicolumn{2}{|c|}{$\mathrm{R} 2=.812$} & \multicolumn{2}{|c|}{$F(55,50)=3.937$} & \multicolumn{2}{|c|}{ Sig. $=.000$} \\
\hline & B & SE B & $B$ & $\mathbf{t}$ & Sig. & R2parcial \\
\hline \multicolumn{7}{|l|}{ Variable } \\
\hline Edad & 1.012 & .417 & .230 & 2.426 & .019 & .104 \\
\hline Lab/Neg & -.280 & .055 & -.478 & -5.109 & .000 & .343 \\
\hline $\begin{array}{l}\text { Imp/IrrpxAtrCre } \\
\text { xgénero }\end{array}$ & .704 & .316 & .784 & 2.229 & .030 & .090 \\
\hline $\begin{array}{l}\text { RegEmxInf.Tris } \\
\text { xgénero }\end{array}$ & 5.041 & 2.567 & .352 & $1.964^{*}$ & .055 & .071 \\
\hline $\begin{array}{l}\text { Grand/Manipx } \\
\text { RegEmxInf.Tris } \\
\text { x género }\end{array}$ & .901 & .441 & .632 & 2.044 & .046 & .077 \\
\hline
\end{tabular}

NOTA: En la tabla sólo se muestran los efectos simples e interactivos significativos y casi significativos - $p<.005$ (")- a $N=106$.

En la tabla Lab/Neg = labilidad / negatividad; Imp//rrpxAtrCrexgénero = interacción entre el rasgo impulsividad / irresponsabilidad, la competencia en atribución de creencia falsa de segundo orden y el género; RegEmxInf.Trisxgénero = interacción entre la competencia en regulación emocional, en inferencia de tristeza de segundo orden y el género; Grand/ Manipx RegEmxInf.Trisx género = interacción entre el rasgo grandlosidad / manipulación, las competencias en regulación emocional y en inferencia de tristeza de segundo orden y el género.

emocionalmente son competentes en inferencia de tristeza de $2^{9}$ orden $(B=-6.133, t=-2.217$, sig. $=.005)$ y se debilita $e$ invierte ligeramente en las chicas poco competentes en regulación y mentalización $(B=-1.166$, $t=-2.120$, sig. $=.025$ ) así como en los chicos poco competentes emocionalmente y con alta competencia en inferencia de tristeza $(B=$ $-1.114, t=-1.276$, sig.=.10). El rasgo conductual de psicopatía predice los niveles más reducidos de éstos -fortaleciendo por lo tanto una relación condicional invertida- en las chicas poco reguladas 
Tabla 4. Resultados de los anállsls de regresión múltiple por el método Introducir de los efectos simples e Interactivos significativos de los rasgos de psicopatía, regulación emocional y teoría de la mente en conducta agresiva. Modelo 3

\begin{tabular}{|c|c|c|c|c|c|c|}
\hline \multicolumn{7}{|c|}{ Conducta Agresiva } \\
\hline \multirow[t]{2}{*}{ Modelo 3} & \multicolumn{2}{|c|}{$\mathrm{R} 2=.848$} & \multicolumn{2}{|c|}{$F(55,50)=5.062$} & \multicolumn{2}{|c|}{ Sig. $=.000$} \\
\hline & $\mathbf{B}$ & SE B & $B$ & $t$ & Sig. & R2parcial \\
\hline \multicolumn{7}{|l|}{ Variable } \\
\hline Edad & 3.580 & 1.186 & .258 & 3.017 & .004 & .153 \\
\hline $\mathrm{Lab} / \mathrm{Neg}$ & -1.362 & .156 & -.738 & -8.747 & .000 & .605 \\
\hline RegEm & .777 & .344 & .300 & 2.262 & .028 & .093 \\
\hline AtrCre & -3.400 & 1.420 & -.273 & -2.394 & .020 & .103 \\
\hline $\begin{array}{l}\text { Grand/Manipx } \\
\text { RegEmxInf.Tri } \\
\text { sxgénero }\end{array}$ & 3.461 & 1.254 & .769 & 2.760 & .008 & .132 \\
\hline
\end{tabular}

NOTA: En la tabla sólo se muestran los efectos simples e interactivos significativos a $\mathrm{N}=106$.

En la tabla Lab/Neg = labilidad / negatividad; RegEm = regulación emocional, AtrCre = atribución de creencia falsa de segundo orden; Grand/ ManipxRegEmxInf.Trisx género = interacción entre el rasgo grandiosidad / manipulación, las competencias en regulación emocional y en inferencia de tristeza de segundo orden y el género.

emocionalmente pero capaces de inferir la inferencia de la tristeza ajena. Estos valores difieren significativamente de los niveles predichos también reducidos en los chicos poco regulados pero capaces de atribuir tristeza $(t=-2.159$, sig.=.025) y en el grupo de chicas poco reguladas y mal lectoras de tristeza ajena de $2^{2}$ orden $(t=-2.402$, sig. =.010), niveles que no difieren significativamente entre sl $(t=-1.079$, no sig.). Por lo tanto, aunque el efecto interactivo se deba fundamentalmente a la vinculación género femenino/alta competencia mentalista en impulsividad, es cierto que los chicos capaces de inferir la inferencia de la tristeza ajena y las chicas que carecen de ésta pueden amortiguar en mayor o menor medida el débil efecto de riesgo del rasgo conductual en síntomas atencionales. 
El modelo 1 también estimó que la combinación InfTris y género en interacción con RegEm tiene capacidad para alterar la relación positiva entre el rasgo Cruel/NoEm y los P.Atención $(B=-8.570)-$ representada en la figura 2- de modo que, como indican la representación de las pendientes simples -su significación y diferencias- el rasgo afectivo de psicopatía se asocia más intensamente con $P$. Atención en las chicas poco competentes emocionalmente que, como en el término anterior, son competentes en inferencia de tristeza de $2^{\circ}$ orden $(B=7.354, t=2.775$, sig. $=.005)$. Es este efecto intensificador de síntomas el que contribuye fundamentalmente a la significación del efecto interactivo, -que explica hasta un $11.6 \%$ de varianza-, dado que además de diferenciarse sigrificativamente de los efectos también intensificadores ( $\mathrm{t}=2.432$, sig.=.010 y $\mathrm{t}=2.497$, sig.=.010) que suceden en los chicos con alta competencia en autorregulación y baja competencia en mentalización ( $B=1.160, t=1.803$, sig.=.025) y en los chicos con baja competencia en las dos $(B=.579, t=1.330$, sign. $=.10$ ), éstos no alcanzan a diferenciarse entre si $(t=.790$, no sig.). Por lo tanto, el rasgo Cruel/NoEm predice los niveles más elevados de P. Atención cuando las chicas poco reguladas son buenas lectoras de la inferencia de la tristeza ajena, prediciendo también, aunque en menor medida, incremento de tales dificultades cuando los chicos menos capaces inferir estados mentales de $2^{\circ}$ orden son más o menos capaces de regularse afectivamente.

Respecto a la C.Delictiva, el modelo 2 estimó que la interacción Atr.Cre y género tiene capacidad para modificar la asociación positiva de la Imp/lrrp con C.Delictiva $(B=.704)$, de forma que, como indican la representación de las pendientes simples y su única significación, este rasgo predice un incremento en el nivel $\mathrm{C}$. Delictiva únicamente para las chicas que, poco competentes en regulación emocional, infieren adecuadamente en otros la inferencia de la falsa creencia ajena $(B=.540, t=2.700$, sig. $=.005)$. Este efecto amplificador de riesgo de la combinación Irnp/lrrp, Atr.Cree y género en sintomatología delictiva -que explica un $9 \%$ de la varianza del criterio- sucede además del efecto directo de $\mathrm{Lab} / \mathrm{Neg}(\mathrm{B}=-.280)$ y edad $(B=1.012)$, que explican un $34.3 \%$ y $10.4 \%$, respectivamente. El efecto también aditivo de la interacción entre Grand/Manip, RegEm, Inf.Tris y género también predice $C$.Delictiva $(B=.901)$, explicando un $7.7 \%$ de su varianza. Los 
análisis de significación de las pendientes simples -y sus diferenciasindican que tal significación refleja fundamentalmente la capacidad amplificadora que la vinculación género femenino / alta competencia emocional / alta competencia inferencial de tristeza $2^{\circ}$ orden ejerce sobre la relación del rasgo Grand/Manip y delincuencia $(B=1.084, t=$ 2.576, sig.=.005). Las chicas más capaces de autorregularse adecuadamente y de atribuir en los otros la inferencia de tristeza ajena tienen una significativamente mayor probabilidad de mostrar conductas delincuentes que las chicas de baja competencia o que los chicos.

Respecto a la C. Agresiva, el modelo 3 estimó como significativos los efectos directos de Lab/Neg $(B=-1.362)$ y género $(B=3.580)$ y los simples de RegEm $(B=.777)$ y de AtrCree $(B=-3.400)$. Lab/Neg y edad predicen C.Agresiva y RegEm en chicos con baja tendencia psicopática y baja competencia mentalista predice C.Agresiva, mientras que AtrCree de $2^{\circ}$ orden en chicos con baja tendencia psicopática y baja RegEm predice menor nivel de C.Agresiva. Además, y al igual que en la explicación de la C.Delictiva, el efecto interactivo de RegEm, InfTris y género tiene la capacidad de modificar la relación positiva Grand/Manip y C.Agresiva $(B=3.461)$, informando de un $13.2 \%$ de su varianza. Dada la representación de las pendientes, su significación y diferencias, la relación entre el rasgo interpersonal y la agresión se hace máxima en las chicas con alta competencia en regulación y en inferencia de tristeza de $2^{\circ}$ orden $(B=2.924, t=2.474$, sig. $=.010)$ y se fortalece en las chicas competentes en inferir tristeza que son poco competentes en autorregulación $(B=.630, t=1.235$, sig. $=.10)$ y en las chicas poco competentes en ambas $(B=.405, t=1.030$, sig.=.10). Los niveles más elevados de agresión predichos por la interacción género femenino, alta RegEm y alta InfTrit difieren significativamente de los niveles, también incrementados, predichos por la interacción género femenino, baja RegEm y alta InfTrist $(t=1.777$, sig. $=.050)$ y por la interacción género femenino, baja RegEm y baja InfTrist $(t=2.261$, sig.=.010), niveles no diferentes entre sí ( $t=.681$, no sig.).

\section{Efectos de mediación}

En los términos especificados por Baron y Kenny (1986), la Lab/ Neg cumple una función de mediación en las relaciones Grand/Manip 
y C.Agresiva. Así, Grand/Manip predijo significativamente variaciones en $\mathrm{Lab} / \mathrm{Neg}[(B=-.259 ; \mathrm{Rp} 2=.038), F(5,122)=11.173]$. La relación entre el rasgo y la $C$.Agresiva fue inicialmente significativa $[(B=.292$; $\mathrm{Rp} 2=.050), F(5,122)=12.992]$ y la Lab/Neg tiene efecto predictor significativo en C.Agresiva controlando género y edad $[(B=-.647 ; \mathrm{Rp} 2$ $=.440), F(6,121)=35.077]$. El porcentaje de varianza del criterio explicado por la mediadora es del $44 \%$ frente al $5 \%$ de la predictora. El efecto predictor del rasgo Grand/Manip en C.Agresiva al introducir en la ecuación el efecto aditivo de Lab/Neg pierde significación [( $B$ $=.124 ; R p 2=.016), F(6,121)=35.077]$, tal que el efecto de mediación de Lab/Neg es completo.

Tabla 5. Resultados de los anállsls de regresión múltiple de los efectos directos e indirectos de los rasgos de psicopatía en conducta agres/va por medio de labllidad / negat/vldad

\begin{tabular}{|c|c|c|c|c|c|c|}
\hline \multicolumn{7}{|c|}{ Conducta Agresiva } \\
\hline Variable & B & SE B & $B$ & $\mathbf{t}$ & Sig. & R2parcial \\
\hline \multicolumn{7}{|c|}{$\begin{array}{l}\text { Regresión de rasgos de psicopatía en Lab/Neg (VD: Lab/Neg, VI: Grand/Manip, } \\
\text { Cruel/NoEm e Imp/Irrp, edad y género) }(\mathrm{R} 2=.314)\end{array}$} \\
\hline Edad & -3.055 & .593 & -.409 & -5.154 & .000 & .178 \\
\hline Género & 3 & 1.027 & .199 & & .016 & .046 \\
\hline Grand/Manip & -.172 & .078 & -.259 & -2 & .029 & .038 \\
\hline \multicolumn{7}{|c|}{$\begin{array}{l}\text { Regresión de rasgos de psicopatia en C. Agresiva (VD: agresión, VI: } \\
\text { Grand/Manip, Cruel/NoEm e Imp/Irrp, edad y género) }(\mathrm{R} 2=.347)\end{array}$} \\
\hline Edad & 5.267 & 1.004 & .406 & 5.247 & .000 & .180 \\
\hline Géner & -3.764 & 1.740 & -.172 & -2.163 & .032 & .036 \\
\hline Grand/Manip & .337 & .132 & .292 & 2.553 & .012 & .050 \\
\hline \multicolumn{7}{|c|}{$\begin{array}{l}\text { Regresión de rasgos de psicopatia y Lab/Neg en C. Agresiva (VD: agresión, VI: } \\
\text { Grand/Manip, Cruel/NoEm e Imp/Irrp, Lab/Neg, edady género) }(\mathrm{R} 2=.635)\end{array}$} \\
\hline Edad & 1.833 & .832 & .141 & 2.203 & .030 & .038 \\
\hline $\mathrm{Lab} / \mathrm{Neg}$ & -1.124 & .115 & -.647 & -9.762 & .000 & .440 \\
\hline
\end{tabular}

NOTA: Para simplificar la presentación, en la tabla únicamente se muestran los efectos significativos en $\mathrm{N}=106$.

En la tabla Lab/Neg = labilidad / negatividad; Grand/Manip = grandiosidad / manipulación; Cruel/NoEm = crueldad/no emocionalidad; $/ \mathrm{mp} / \mathrm{mp}=\mathrm{impulsividad}$ / irresponsabilidad; C.Agresiva = conducta agresiva . 


\section{Efectos de moderación mediada}

En ninguno de los modelos de regresión múltiple estimados se obtuvieron efectos de moderación mediada.

\section{DISCUSIÓN Y CONCLUSIONES}

El objetivo fundamental de este trabajo es el esclarecimiento de cómo los rasgos conductual, afectivo e interpersonal de psicopatía, labilidad / afectividad negativa, regulación emocional, teoría de la mente en su multicomponencialidad y género se relacionan específicamente para explicar heterogeneidad conductual en adolescentes normales de 12 a 15 años en contexto escolar. En general, los resultados plantean tanto un efecto aditivo como un efecto interactivo de las competencias emocional y mentalista y del género específicamente con cada uno de los rasgos psicopáticos en la predicción de las diferentes formas de desadaptación y adaptación externalizante. Es decir, la naturaleza de las relaciones rasgos psicopáticos / problemas atencionales, agresión y delincuencia tienen una cualidad diferencial en función del estatus emocional, mentalista y genérico del adolescente, independientemente de las contribuciones únicas y directas que los componentes temperamental y aprendido de regulación emocional y de teoría de la mente pudieran hacer.

Como era de esperar dada la condición no clínica de la muestra, los niveles de funcionamiento psicológico y comportamental evaluados están dentro del rango normativo. El perfil de relaciones no sólo muestra una co-ocurreencia entre rasgos sino además una asociación positiva y "diferencial" de éstos con sintomatología y emocionalidad. Sorprendentemente, dada la literatura que vincula el rasgo afectivo con problemas de conducta a un patrón de conducta antisocial severa y agresiva (Andershed, Kerr y Stattin, 2002; Frick y cols., 2005), y coincidiendo, no obstante, con trabajos en los que tal rasgo está débilmente asociado con sintomatología al controlar el efecto de los restantes (Frick y cols., 2000), son los rasgos interpersonal y conductual los que mantienen una relación significativa con inatención, agresión y delincuencia, además de con labilidad/negatividad, mientras que el afectivo únicamente lo hace con problemas atencionales. Este 
perfil de relaciones plantea la necesidad de considerar y contrarrestar el efecto individual y conjunto de las tres dimensiones psicopáticas en la explicación de los problemas de conducta y de su operación respecto a los potenciales mecanismos y procesos de riesgo y protección.

Respecto al primer objetivo, la hipótesis "1" se confirma parcialmente dado que los resultados muestran cómo labilidad emocional y afectividad negativa es uno de los mecanismos que explican la capacidad predictiva del rasgo grandiosidad/manipulación en conducta agresiva adolescente además del efecto aditivo significativo del género y edad y no significativo de los rasgos afectivo y conductual. De hecho, el hallazgo del efecto de mediación completa plantea que es este componente temperamental de regulación emocional el mecanismo fundamental en la actualización del rasgo interpersonal. La expresividad y responsividad emocional negativa e inadecuada en las relaciones con iguales -ante propuestas sociales que probablemente interpreta erróneamente como un desafío a su autoestima así como para generar respuestas sociales que reduzcan su angustia-, la baja tolerancia a situaciones emocionales, la débil recuperación y control atencional e inhibitorio en episodios emocionales y la labilidad $\theta$ inflexibilidad emocional son la base sobre la que se asientan los comportamientos psicopáticos y que, asimismo, contribuyen a vulnerabilidad y explican desadaptación expresada en conducta agresiva. A su vez, tales comportamientos narcisistas, considerados procesos autorregulatorios disfuncionales que sirven al mantenimiento de la, resultan en y mantienen el afecto negativo (Morf and Rhodewalt, 2001), responsable de la conducta agresiva. Tal afecto negativo conduciria a una vía circular en la que genera un estilo atribucional y de afrontamiento sesgado $\theta$ inadecuado (Lengua y Long, 2002), mediador de la reactividad emocional negativa (Rhodewalt y Morf, 1998).

Este resultado, que es consistente con los trabajos que demuestran la capacidad predictiva de la negatividad, labilidad e infracontrol voluntario en conducta externalizante (Eisenberg y cols., 2005; Loney, Lima y Butler, 2006; Olson, Sameroff, Kerr, López y Wellman, 2005), apunta a su papel mediador en la actualización del rasgo interpersonal de psicopatía. Apoya así el carácter de riesgo de la dimensión narcisista además de la afectiva (Barry y cols., 2003) y especifica el mecanismo que informa de tal riesgo. Queremos señalar también, 
considerando la función moderadora de la autoestima en la actuación de dicha dimensión (Barry y cols., 2003) y en la medida en que los comportamientos autorregulatorios narcisistas están al servicio de la autoestima, que posteriores trabajos podrían considerar modelos de moderación mediada en los que se contemplen estos procesos, lo que contribuiría a comprender mejor esta trayectoria.

Paralelamente, y dado el alto porcentaje de agresión explicado por labilidad / negatividad, el resultado indica que el componente temperamental de la regulación emocional representa un mecanismo clave en la emergencia de la conducta antisocial (Olson y cols., 2005). No obstante, y aun cuando ningún otro rasgo haya explicado sintomatologla mediada por labilidad, el rasgo interpersonal responde, solo en un grado mínimo, de agresión y de labilidad / negatividad.

Contrario a lo esperado, tenlendo en mente la asociación positiva del rasgo conductual de psicopatía con conducta antisocial $\theta$ inestabilidad emocional / afectividad negativa (Frick y cols., 1999; Lynam, Caspi, Moffit, Raine, Loeber y Stouthamer-Loeber, 2005), los resultados indican que labilidad afectiva y emocionalidad negativa no explica la asociación referida entre el rasgo y la sintomatología. Esta ausencia de relación, que también ha observado Lima (2004), podría, tal vez; atribuires a los componentes temperamentales de regulación emocional del rasgo. El componente de impulsividad al igual que los de irresponsabilidad y búsqueda de emociones conllevan control reactivo (Martel y Nigg, 2006) y no control voluntario ni responsividad afectiva -contemplados en el ERC-, y los dos últimos implican, también, nivel de arousal reducido (Bradley, 2000). Dado que las dificultades en control voluntario -o inatención- y reactivo -0 impulsividad-y la emocionalidad negativa, que hacen contribuciones únicas y aditivas a la emergencia de problemas de conducta (Eisenberg y cols., 2001, 2005; Lengua, 2003; Martel y Nigg, 2006), así como que el TDAH no se asocie al perfil temperamental de reactividad emocional elevada, labilidad e inflexibilidad emocional a no ser que concurran con agresión (Melnick y Hinshaw, 2000) resulta lógico que los componentes impulsividad/búsqueda de emociones no predigan emocionalidad o labilidad emocional aunque si desinhibición_reactiva.

Respecto al rasgo afectivo de psicopatía, y también contrario a lo esperado en vista de su relación con reactividad reducida a 
estimulación emocional negativa (Loney y cols., 2003) -particularmente amenazadora y de tristeza- (Blair, 1999), y baja negatividad (Frick y cols., 1999) asi como al posible papel mediador de arousal reducido en el temperamento desinhibido (Raine, Reynolds, Venables, Mednick y Farrington, 1998), la labilidad/afectividad negativa no opera como un mecanismo explicativo de desadaptación conductual. Nuestro resultado es consistente con el obtenido por Lima (2004) respecto a la ausencia de relación controlada entre rasgo afectivo y negatividad / responsividad emocional. La ausencia de relaciones podría deberse, al menos en parte, a las características de la evaluación que incluyen medidas de regulación emocional de carácter general y no específicamente centradas en reactividad emocional a amenaza y tristeza. En consecuencia, dada la reducida reactividad emocional al miedo y a la tristeza implicada en el rasgo crueldad/insensibilidad y a las asociaciones inconsistentes de éste con afectividad negativa (Frick y cols., 1999; Lima, 2004), cabría la posibilidad de que afectividad negativa ejerza un papel moderador conjunto con afectividad positiva (Loney y cols., 2006) y control voluntario sobre el efecto mediador de la reactividad emocional a dicha estimulación.

En relación al segundo, tercer y cuarto objetivos, los resultados apoyan la necesidad de tener en mente modelos de moderación si de lo que se trata es de acercarnos a comprender el efecto diferencial de los rasgos psicopáticos en desadaptación conductual. En este sentido, y aunque en nuestro estudio sólo se confirmen parcialmente las hipótesis planteadas, la intensidad e incluso dirección del efecto de cada uno de los rasgos difiere en función de la interdependencia regulación emocional e inferencia de los estados emocional y cognitivo. Además, tal efecto interactivo -de protección a veces pero fundamentalmente de riesgo- es específico en función del género.

En concreto, la alta regulación emocional y el adecuado conocimiento de la inferencia de tristeza ajena se asocia con un incremento de síntomas delictivos $y$, en especial, agresivos en las chicas con elevado rasgo interpersonal de psicopatía. Esto es, y contrario a la hipótesis $2 b$ en relación con este rasgo, las chicas con tendencia a un sentido grandioso del yo y a la manipulación de los otros que, además de ser buenas lectoras de la angustia ajena, son conocedoras de sus propias emociones y capaces de modular su expresión 
emocional de manera socialmente adecuada tienen mayor probabilidad de manifestar conducta delictiva y agresiva en el contexto educativo. A su vez, las chicas reguladas emocionalmente que no son capaces de inferir la inferencia de los estados emocionales en los demás -es decir, que no tienen un desarrollo normativo de la teoría de la mente- no tienden a mostrar conducta antisocial ni agresiva, por lo que es la acción conjunta entre competencia emocional y mentalista -respecto a la tristeza- la que determina el peor pronóstico en las chicas adolescentes. No obstante, y a diferencia de lo que ocurre en las chicas, la regulación emocional y la inferencia de tristeza se asocian a una reducción, aunque no significativa estadísticamente, en el nivel de conducta delictiva y agresiva en los chicos con tendencia a la grandiosidad / manipulación, lo que supone una actuación diferencial de los procesos regulatorios y mentalistas emocionales. A este respecto, y aun cuando el rasgo interpersonal no es un indicador de especial severidad en problemas de conducta como es el rasgo afectivo, este hecho diferencial en el que la normalidad en el funcionamiento de la regulación emocional y la mentalización constituye, frente a lo que cabría esperar (Masten, 2001), un factor de riesgo especialmentre relevante en la adolescencia, sugiere que prácticas de socialización genericamente diferentes dadas las presiones y expectativas sociales (Keenan y Shaw, 1997) -donde se le enseña a las chicas a atender y a responder a los estados mentales de los otros- pueden dar cuenta de la mayor regulación emocional y toma de perspectiva socioemocional de las chicas $y$, posiblemente, favorecer una imagen personal dependiente de las relaciones sociales y de los otros (ver Odgers y Moretti, 2002). Esta posibilidad concuerda con los resultados de Vitale, Newman, Bates, Goodnight, Dodge y Pettit (2005) respecto a la mayor capacidad regulatoria del comportamiento desinhibido en chicas con tendencia psicopática global y déficits en procesos de inhibición automática. Las chicas con tendencia a la grandiosidad han podido haber aprendido habilidades regulatorias y mentalistas adecuadas social y culturalmente y además muy útiles de cara a sus objetivos interpersonales particulares -es decir, la manipulación de y la mentira a los otros, el atractivo deshonesto de cara al mantenimiento de su autoestima- que, lejos de amortiguar, les facilita especialmente la 
comisión de actos delictivos y agresivos posiblemente más sutiles, encubiertos y relacionales.

No obstante, la dificultad en auto-regulación emocional continúa asociándose con un incremento en conducte agresiva $=-s i$ bien significativamente menor- en aquellas chicas con tendencia narcisista conocedoras de la inferencia de la tristeza ajena. Aunque predecible en el contexto de las aproximaciones teóricas que consideran en riesgo de desarrollar conducta antisocial al narcisista como no regulado afectivemente (Strauman, 2001) y al buen lector mental bajo condición de no responsivided empática (Hoffman, 1975), los resul= tados muestran cómo ese efecto intensificedor no se diferencia significativamente del ejercido en las chicas no reguladas emocionalmente que no son capaces de inferir tristeza. En consecuencia, parece que to importante para las chicas con tendencia narcisista no reguladas es la disfuncionalidad en autorregulación emocional más que el nivel de competencia mentalista. Además, y también contrario a lo esperado, el efecto de esta interdependencia rasgo interpersonal, competencias emacional y mentalista y génerono es explicada por el mecanismo modiador labilidad / negativided.

En este sentido, y teniendo en mente que estos efectos intensificadores de riesgo suceden en las chicas adolescentes y no, sin embargo, en los chicos -a diferencia del efecto mediador de labilidad amocional y afectividad negativa que ocurre al controlar género- y que la valoración de la conducta antisociad y agresiva es generatizada respecto a la entisocial, abierta y encubierta, y a la agresiva, reactiva y preactiva, asociadas diferencial y respoctivemente a alta / baja negatividad y a no reguleción / regulación emocional (ver Mullin y Hinshaw, 2006)-, estos detos podrían estar sugiriendo la presencia de diversas vias a través de las cuales el rasgo grandiosidad / manipulación en chicas se expresa en problemas de conducta en la etapa adolescente. Además, en la medida en que la autoestima modera la capacidad predictiva del narcisismo (Barry y cols., 2003), cada una de estas vias podría implicar un nivel - cualidad de autoestima diferente, de tal manera que fuera ésta organizadora de estas diferencias en moderación -respecto a regulación y no rogulación emocional- e inconsistencias con mediación -respecto a regulacion emocional y labilidad/negatividad. Asi, los 
adolescentes con mayor probabilidad de manifestar conducta delictiva y agresiva serian las chicas que, conscientes de la angustia del compañero o adulto en situación de "sorpresa desagradable" muestran una emocionalidad y respuesta emocional adecuada en situaciones de interacción social, de forma que es posible que los comportamientos narcisistas traducidos en manipulación, mentira y atractivo deshonesto -que asimismo reflejan competencia regulatoria y mentalista- puedan responder a una autoestima alta y seguridad en si misma $-y$, consiguientemente a una menor vulnerabilidad temperamental-. Paralelamente, habría un grupo de adolescentes cualitativamente diferentes que estarian en riesgo -aunque menor- de desarrollar un comportamiento agresivo, y posiblemente también cualitativamente diferente es decir, reactivo más que proactivo. Serian aquellas chicas que no son capaces de controlar y modular de manera socialmente adecuada sus estados y reacciones emocionales ante propuestas o actos de los otros -posiblemente malinterpretados como amenazantes-, de manera que en estos casos se podría pensar que el comportamiento narcisista pudiera responder a una autoestima idealizada pero frágil. Serian estos los casos en los que la labilidad/ negatividad podría clarificar et efecto interactivo de riesgo

En el rasgo afectivo de psicopatía se observa un perfil similar a esta posible via. La dificuttad en regulación emocional y la adecuada inferencia de la tristeza ajena de segundo orden se asocian a un incremento importante en prablemas atencionales en las chicas adolescentes que informaron de una elevada tendencia a la crueldad / no emocionalidad. Se confirmó la hipotesis $2 b$ en relación con este rasgo; es decir, en las chicas que tienden a ser insensibles y crueles respecto a los estados desagradables de los otros asi como a no sentir angustia - culpabilidad por las consecuencias negativas que au comportamiento tiene en el otro y que, además, muestran deficit en conocimiento y regulación emocional, la habilidad para inferir adecuadamente la angustia y tristeza en los otros las hace especialmente proctives a desarrollar y manifestar sintomatología atencional en el contexto escolar. Merece destacarse que el único efecto interactivo que parece capaz de reducir sintomatología atencional, aunque no alcance significación estadistica, es la ausencia de regulación emocional junto 
con ausencia de inferencia de tristeza ajena en chicas, lo que sugiere que es esta competencia mentalista uno de los procesos socioemocionales que juegan un papel fundamental en la actualización e inhibición del rasgo afectivo de psicopatía. Los niños y adolescentes con rasgos de crueldad / insensibilidad tienden a ser hipo-responsivos o no reaccionan psicofisiológica y afectivamente a las expresiones faciales de angustia o tristeza en los compañeros o adultos (Blair, 1999; Blair y cols., 1997). Es decir, no experimentan arousal incrementado $o$ angustia por la tristeza de los otros independientemente de cual sea su causa por lo que la atribución y conocimiento de esa tristeza en situación estereotipada imaginada $-y$ no de otros estados como el enfado- no hace sino acrecentar en un grado máximo el efecto negativo de la tendencia a la insensibilidad y crueldad en desadaptación y, sin embargo, no frena tal comportamiento desinhibido como cabría esperar bajo condiciones normativas de reactividad a señales de angustia o miedo ajeno (Blair, 1995). Este efecto observado en la tendencia a la psicopatía en chicas supone así un apoyo indirecto al modelo neurocognitivo del Mecanismo de inhibición a la Violencia (Blair, 2001; Blair y Frith, 2000) en la medida en que la identificación de la tristeza, y no del enfado ajeno, inferida de una situación de sorpresa desagradable imaginada intensifica el efecto del rasgo crueldad / insensibilidad en jóvenes sin problemas de conducta puesto que son insensibles a la connotación aversiva o de castigo asociada al arousal incrementado que tiene el dolor ajeno interfiriendo así en una socialización moral adecuada. Además, dicho efecto en jóvenes con mayor nivel de rasgo afectivo de psicopatía concuerda con la incapacidad para beneficiarse de las prácticas de socialización basadas en la inducción de empatía en niños con tendencia psicopática (Wooton, Frick, Shelton y Silverthorn, 1997) y señala, asimismo, la importancia de integrar el perfil temperamental del joven con tendencia afectiva a la psicopatía en el diseño programas educativos (Hawes y Dadds, 2005). Hay que tener en cuenta además que las dificultades temperamentales, los deterioros neurocognitivos, los rasgos de personalidad como el de crueldad y la impulsividad están diferencialmente asociados a la trayectoria de inicio temprano $y$, por otro lado, el conocimiento de iguales delincuentes lo está a la trayectoria de inicio tardío (Moffitt, 1993). Por consiguiente, nuestro resultado en la medida 
en que, en la población adolescente, son las chicas con mayor nivel de rasgos afectivos las que bajo el efecto de determinados factores de riesgo posiblemente "claves" para el desarrollo de problemas de conducta -poco reguladas emocionalmente pero conocedoras de la inferencia del dolor ajeno- tienen una mayor probabilidad de manifestar dificultades atencionales -deterioro neurocognitivo asociado inconsistentemente con psicopatía (Blair, Mitchel y Blair, 2005) e implicado en el proceso de "evitación pasiva" (Newman, 1998) deteriorado a su vez en población con tendencia psicopática (Vitale y cols., 2005). El resultado coincide así en términos de riesgo con los que subyacen al trastorno de conducta siguiendo la "vía de comienzo demorado" (Silverthorn y Frick, 1999) que, a su vez, son coincidentes con los que señalan la trayectoria evolutiva al trastorno disocial en niños.

Por otro lado, este efecto fortalecedor, consistente con el hallazgo de Sutton y cols. (2000) respecto a la dimensión "ausencia de remordimiento", apoya la "teoria de las mentes malignas" de Happé y Frith (1996) así como las observaciones de Repacholi y Slaughter (2003) y Sutton y cols. (1999) de que muchos "bullies" pueden ser buenos mentalistas ante objetivo antisocial ya que, bajo condiciones psicológicas específicas -rasgo afectivo de psicopatía y consiguiente pobre regulación emocional-, la inferencia del estado de tristeza ajena inferido por otro en situación de sorpresa desagradable (el heladero no está donde le dijo que estaría y sabiendo que él quería comprarle un helado) conlleva un mayor riesgo de manifestar sintomatología externalizante.

Respecto a los chicos normales con mayor tendencia afectiva a la psicopatía, a diferencia de cómo operan simultáneamente los procesos regulatorios y mentalistas en las chicas con mayor tendencia psicopática, se observa que la alta regulación y la baja regulación emocional se asocian a un mayor y menor incremento de dificultades atencionales en baja competencia mentalista. Esto es, los chicos que informaron de mayor tendencia a la crueldad e insensibilidad a los estados mentales y necesidades de los demás y que desarrollaron una habilidad adecuada para manejar y modelar sus respuestas emocionales en situaciones sociales tienen mayor probabilidad de manifestar inatención cuando no son capaces de 
inferir correctamente los estados emocionales y cognitivos ajenos que aquellos no hábiles en autorregulación emocional, los cuales también tienen una probabilidad alta de manifestar inatención. A diferencia de lo que sucede en las chicas, para ninguno de estos casos el proceso mentalista de atribución de tristeza ajena tiene un papel relevante en la actualización o inhibición del rasgo psicopático. Incluso, la baja competencia regulatoria y mentalista ejerce un efecto contrario en la relación tendencia psicopática / inatención en función del género; esto es, este efecto interactivo que fortalece el efecto del rasgo psicopático en chicos amortigua -aunque no significativamenteel efecto del mismo rasgo en chicas. Además, las probabilidades de incremento sintomático observadas en chicos son significativamente menores que la observada en chicas. Por lo tanto, estos resultados no concuerdan, al menos por lo que se refiere a los factores de riesgo implicados en cada una de las dos trayectorias evolutivas clásicas a los problemas de conducta, con la propuesta de equivalencia genérica de Moffitt y Caspi (2001). En cambio si concuerdan con la diferencia en trayectorias al trastorno disocial en función del género planteada por Silverthorn y Frick (1999).

Aun cuando este resultado sea relativamente inconsistente con trabajos actuales en los que se ha demostrado que el rasgo crueldad / insensibilidad predice conducta antisocial y agresiva en chicos y chicas (Marsee, Silverthorn y Frick, 2005; Pardini, Obradovic y Loeber, 2006), el hecho de que, aun en una muestra adolescente no clínica, el rasgo crueldad / insensibilidad de psicopatía, bajo condiciones socio-emocionales y cognitivas especificas, se asocie al mayor incremento en sintomatología externalizante -junto con el rasgo grandiosidad / manipulación- y que esta sintomatología se exprese en dificultad atencional, que es predictor de conducta antisocial y agresiva (Pardini y cols., 2006) y sirve como un marcador conductual indirecto de deterioros neurocognitivos implicados en el desarrollo de los rasgos psicopáticos (Moffitt, 1993; Newman, 1998), parece sugerirnos la importancia que tienen los rasgos afectivos en la identificación de una via severa a los problemas de conducta (Barry y cols., 2000).

Paralelamente al efecto diferencial del rasgo afectivo en chicas en función del nivel de competencia regulatoria y mentalista, resulta especialmente interesante que la baja regulación emocional y la alta 
inferencia de tristeza ajena de $2^{2}$ orden ejerzan conjuntamente el efecto contrario respecto al rasgo conductual de psicopatía. Parcialmente coherente con la hipótesis $2 \mathrm{~b}$ correspondiente, y reflejando así de nuevo la importancia de considerar la multicomponencialidad de cada uno de los constructos o procesos implicados en el funcionamiento humano, la capacidad para inferir el estado de tristeza ajena junto con la dificultad en autorregulación emocional logra invertir el efecto de riesgo del rasgo conductual de psicopatía asociándose a una reducción muy significativa de sintomatología atencional en las chicas con mayor tendencia a la impulsividad/irresponsabilidad. Es, por lo tanto, -y contrario a lo que sucede respecto al rasgo afectivo- el saber cuándo los compañeros se sienten tristes $-\mathrm{y}$ posiblemente el experimentar y sentir angustia por esa tristeza (Pardini y cols., 2003)- lo que dificulta, interrumpe e inhibe el despliegue de esa respuesta -impulsiva e irresponsable- (Blair, 1995) no reflexionada e incentivada favoreciendo, consiguientemente, la actuación de sistemas de control deliberados como es el atencional. Éste, como proceso esencial en el funcionamiento de los diversos niveles autoregulatorios (Calkins y Fox, 2002), favorecería la adaptación comportamental de la adolescente en las diversas situaciones interpersonales y escolares en la medida en que facilita la adecuabilidad del sistema cognitivo, emocional, atencional y conductual.

Es interesante destacar que una misma habilidad como es la atribución de la inferencia de tristeza en los otros puede ejercer efectos diferentes e incluso opuestos. Así funcionaría como factor de riesgo en las chicas poco reguladas con mayor tendencia a la crueldad $e$ insensibilidad y como factor de protección en las chicas poco reguladas con mayor tendencia a la impulsividad e irresponsabilidad respecto a los problemas atencionales. Parece pues que son las diferencias individuales en reactividad al entorno, que comprometen los rasgos de personalidad (Rothbart y Bates, 1998) -responsividad emocional empática-, las responsables de tal discrepancia. Diferencias en reactividad emocional pueden explicar diferencias en el desarrollo de la preocupación empática por los otros (Blair, 1999; Kochanska, 1993) por lo que, constantes tales diferencias en reactividad, cabe pensar que el conocimiento de la angustia en el otro consiga realzar las diferencias en preocupación empática. Por otro 
lado, la ausencia de mediación temperamental -como se ha explicado- podría deberse a la inespecificidad de su evaluación. Estos resultados de moderación son, a su vez, coherentes con los trabajos de Davis (1994) y Hastings y cols. (2000) respecto al papel protector de la empatía y de la preocupación por los otros en los problemas externalizantes ya que tanto la empatía como la preocupación por los otros implican no sólo el conocer el estado emocional ajeno sino además el compartirlo o reaccionar afectivamente en consecuencia. Como Patrick (1994) afirmó, los psicopatas pueden conocer la pena en los demás pero no se sienten motivados por ella. Por lo tanto, cabe pensar que para el desarrollo de la empatía no sólo se requiere de una regulación emocional adecuada además de la habilidad de mentalización sino también, y fundamentalmente, de una responsividad emocional o empática a los estados emocionales y cognitivos de los demás -esto es, de la experiencia de tristeza (ver Eisenberg, 2000).

Resulta interesante además que la combinación baja regulación y capacidad de atribuir la inferencia de la tristeza ajena también resulta en amortiguación de dificultades atencionales, aunque en significativo menor grado, en los chicos que informan de mayor tendencia a la impulsividad e irresponsabilidad de tal manera que y a diferencia de lo que sucede en los rasgos afectivo e interpersonal de psicopatía- la operación diferencial de los procesos socio-emocionales de protección es similar tanto en chicos como en chicas, ejerciendo aun así el género femenino un papel significativamente más protector de desadaptación atencional. Esto es, y a diferencia de los restantes factores de riesgo de los rasgos interpersonal y afectivo de psicopatía, hay equivalencia genérica adolescente en el factor de protección del rasgo de psicopatía vinculado al trastorno de personalidad antisocial.

Por otro lado, la capacidad de atribuir adecuadamente la inferencia de creencia falsa en situación de sorpresa desagradable se asocia a incremento del nivel de conducta delincuente en chicas impulsivas e irresponsables y poco reguladas emocionalemnete. En este sentido, y contrano a la hipótesis, la competencia en mentalización cognitiva sitúa en riesgo a las chicas con tendencia conductual a la psicopatía. En chicos no se observa ni siquiera la misma tendencia de relación. Quizás el mecanismo responsable de este efecto fortalecedor de 
riesgo tenga que ver, más que con procesos implicados en el desarrollo de la empatía, con sesgos en el procesamiento de información social como por ejemplo, la tendencia a atribuir intención hostil a los otros- como se observó en niños y en chicos adolescentes con problemas de conducta sin rasgo afectivo psicopático (Frick y cols., 2003).

En conclusión, los resultados indican que cada una de las dimensiones de psicopatía autoinformadas por adolescentes sin problemas de conducta son relevantes en la explicación de la sintomatología externalizante observada en el contexto educativo en consonacia con los hallazgos de Marsee y cols. (2005) en una muestra comunitaria con adolescentes de ambos sexos. Además, ampliando así los hallazgos previos en muestra con adolescentes comunitarios con problemas de conducta y rasgos afectivos, interpersonales $y$ conductuales (Barry y cols., 2003; Frick y cols., 2003; Frick y cols., 2005) y con especial relevancia de cara a los desarrollos teóricos y de promoción de programas de prevención e intervención, los resultados destacan la centralidad de labilidad emocional y afectividad negativa en la explicación de la actualización del rasgo interpersonal de psicopatia y que las diferencias individuales en regulación emocional y conocimiento de la inferencia de tristeza ajena, dependientes de género, alteran la dirección e intensidad de la capacidad operativa de cada una de las dimensiones psicopáticas. Específicamente, los resultados subrayan como factores de riesgo la interdependencia alta competencia en regulación emocional y en inferencia de tristeza respecto a la actuación del rasgo interpersonal en chicas y la interdependencia baja competencia en regulación emocional y alta competencia en inferencia de tristeza respecto a la actuación de los rasgos afectivo e interpersonal en chicas. Destacan, a su vez, como factores de protección la interdependencia baja competencia regulatoria y alta competencia en inferencia de tristeza respecto a la actuación del rasgo conductual en chicas. Así, las diferencias de género se reflejan tanto en el nivel de problemas de conducta informados como en el modo en el que operan las interacciones de los procesos regulatorios y mentalistas en la actualización o inhibición del efecto de los rasgos de psicopatía (Odgers y Moretti, 2002). En este sentido, este trabajo, en el que se han considerado las limitaciones referidas en el estudio de los rasgos psicopáticos en población 
infanto-juvenil (Marsee y cols., 2005), aporta un perfil complejo de relaciones moderadas por procesos emocionales y genéricos entre los rasgos y los problemas de conducta. Por lo tanto, para el esclarecimiento y la comprensión de las diversas trayectorias evolutivas a los problemas de conducta y al funcionamiento adaptativo o resistencia en la en la etapa preadolescente de 12 - 15 años- parece esencial la consideración de disposiciones, habilidades socio-emocionales y condicionamientos que el género pueda imponer sobre las mismas.

\section{REFERENCIAS BIBLIOGRÁFICAS}

Achenbach, T.M. (1991). Manual for Teacher's Report Form and 1991 profile. Burlington, Vt: University of Vermont.

Aiken, L.S. y West, S.G. (1991). Multiple regression: Testing and interpreting interactions. Newbury Park, London: Sage.

Andershed, H., Kerr, M. y Stattin, H. (2002). Understanding the abnormal by studying the normal. Acta Psychiatric Scanndinavian, 106, 7580.

Andershed, H., Kerr, M., Stattin, H. y Levander, S. (2002). Psychopathic traits in non-referred youths: Initial test of a new assessment tool. En E. Blaauw y L. Sheridan (Eds.), Psychopaths: Current International Perspectives (pp.131-158). The Hague: Elseiver.

Astington, J.W. (2003). Sometimes necessary, never sufficient: Falsebelief understanding and social competence. En B. Repacholi y V. Slaughter (Eds.), Individual differences in theory of mind (pp.1338). New Jork: Psychological

Baron, R.M. y Kenny, D.A. (1986). The moderator-mediator variable distinction in social psychological research: Conceptual, strategic and statistical considerations. Journal of Personality Social Psychology, 51(6), 1173-1182.

Barry, C.T., Frick, P.J. y Killian, A.L. (2003). The relation of narcissism and self-esteem to conduct problems in children: An preliminary investigation. Journal of Clinical Child and Adolescent Psychology, 32(1), 139-152.

Barry, C.T., Frick, P.J., DeShazo, T.M., McCoy, M.G., Ellis, M. y Loney, B.R. (2000). The importance of callous-unemotional traits of 
extending the concept of psychopathy to children. Journal of Abnormal Psychology, 109, 335-340.

Blair, K.A., Denham, S.A., Kochanoff, A. y Wipple, B. (2004). Playing it cool: Temperament, emotion regulation and social behavior in preschoolers. Journal of School Psychology, 42, 419-443.

Blair, R.J.F., Colledge, E., Murray, L. y Mitchell, D.G.V. (2001). A selective impairment in the processing of sad and fearful expressions in children with psychopathic tendencies. Journal of Abnormal Child Psychology, 29, 491-498.

Blair, R.J.F., Mitchel, D.G.V. y Blair, K.A. (2005). The psychopath: Emotion and the brain. Malden, U.S.: Blackwell Publishing.

Blair, R.J.R. (1995). A cognitive developmental approach to morality: Investigating the psychopath. Cognition, 57, 1-29.

Blair, R.J.R. (1999). Responsiveness to distress cues in the child with psychopathic tendencies. Personality and Individual Differences, $27,135-145$.

Blair, R.J.R. y Frith, U. (2000). Neurocognitive explanations of the antisocial personality disorders. Criminal Behaviour and Mental Health, 10, 566-582.

Bradley, S.J. (2000). Affect regulation and the development of psychopathology. New Jork: Guilford Press.

Buitelaar, J.K., Van Der Wess, M., Swaab-Barneveld, J.T. y Jan Van Der Gaga, R.J. (1999). Theory of mind and emotion-recognition functioning in autistic spectrum disorders and in psychiatric control and normal children. Development and Psychopathology, 11, 3958.

Calkins, S.D. y Fox, N.A. (2002). Self-regulatory processes in early personality development: A multilevel approach to the study of childhood social withdrawal and aggression. Development and Psychopathology, 14, 477-498.

Campos, J.J., Frankel, C.B. y Camras, L. (2004). On the nature of emotion regulation. Child Development, 75(2), 377-394.

Caspi, A., Moffitt, T.E., Newman, D.L. y Silva, P.A. (1998). Behavioral obsenvations at age 3 years predict adult psychiatric disorders: Longitudinal evidence from a birth cohort. En M. Hertzig y E. Farber (Eds.), Annual progress in child psichiatry and child development (pp.319-331). Philadelphia, PA: Brunner. 
Cicchetti, D., Ackerman, B.P. y Izard, C.E. (1995). Emotions and emotion regulation in developmental psychopathology. Development and psychopathology, 7, 1-10.

Clecklely, H.C. (1941). The mask of sanity: An attempt to reinterpret the so-called psychopathic personality. St. Louis, MO: Mosby.

Cliffordson, C. (2002).The hierarchical structure of empathy: Dimensional organization and relations to social functioning. Scandinavian Journal of Psychology, 43, 49-60.

Cole, P.M., Michel, M.K. y Teti, L.O. (1994).The development of emotion regulation and dysregulation: $A$ clinical perspective. Monographs of the Society for Research in Child Development, 59 (Serial №.240), 73-100.

Colledge, E. y Blair, R.J.R. (2001). The relationship in children between the inattention and impulsivity components of attention deficit and hyperactivity disorder and psychopathic tendencies. Personality and Individual Differences, 30, 1175-1187.

Cutting, A. y Dunn, J. (2002). The cost of understanding other people: Social cognition predicts young children's sensitivity to criticism. Journal of Child Psychology and Psychiatry, 43, 849-860.

Davis, M.H. (1994). Empathy: A social psychological approach. Colorado: Westview.

Dawson, J. F. \& Richter, A. W. (2006). Probing three-way interactions: The development and application of a slope difference test. Journal of Applied Psychology, 91, 917-926.

Decety, J. y Jackson, P.L. (2004). The functional architecture of human empathy. Behavioral and Cognitive Neuroscience Reviews, 3(2), 71-100.

Denham, S.A. (1986). Social cognition, social behavior and emotion in preschoolers: Contextual validation. Child Development, 57, 194201.

Eisenberg, N. (2000). Emotion, regulation and moral development. Annual Review in Psychology, 51, 665-697.

Eisenberg, N. y Morris, A.S. (2002). Children's emotion's related regulation. En R. Kail (Ed.), Advances in child development and behavior (Vol.30, pp.190-229). San Diego, CA: Ámsterdam.

Eisenberg, N., Cumberland, A., Spinrad, T.L., Fabes, R.A., Shepard, S.A., Reiser, M., Murphy, B.C., Losoya, S.H. y Guthrie, I.K. (2001). 
The relations of regulation and emotionality to children's extemalizing and internalizing problems behavior. Child Development, 72(4), 1112-1134.

Eisenberg, N., Fabes, R.A., Guthrie, I.K. y Reiser, M. (2002). The role of emotionality and regulation in children's social competence and adjustment. En L.Pulkkinen y A. Caspi (Eds.), Paths to sucessful development: Personality in the life course (pp. 46-70). New Jork: Cambridge University.

Eisenberg, N., Sadovsky, A., Spinrad, T.L., Fabes, R.A., Losoya, S.H., Valiente, C., Reiser, M., Cumberland, A. y Shepard, S.A. (2005). The relations of problem behavior status to children's negative emotionality, effortful control and impulsivity: Concurrent relations and prediction of change. Developmental Psychology, 41(1), 193-211.

Eisenberg, N., Spinrad, T.L., Fabes, R.A., Reiser, M., Cumberland, A., Shepard, S.A., Valiente, C., Losoya, S.H., Guthrie, I.K. y Thompson, M. (2004). The relations of effortful control and impulsivity to children's resiliency and adjustment. Child Development, 75(1), 25-46.

Fahie, C.M. y Symons, D.K. (2003). Executive functioning and theory of mind in children clinically referred for attention and behavior problems. Applied Developmental Psychology, 24, 51-73.

Feshbach, N. (1987). Parental empathy and child adjustment/ maladjustment. En N. Eisenberg y J. Strayer (Eds.), Empathy and its development (pp.271-291). Cambridge, England: Cambridge University Press.

Frick, P.J. (2001). Effective interventions for children and adolescents with conduct disorder. The Canadian Journal of Psychiatry, 46, 2637.

Frick, P.J. (2004). Developmental pathways to conduct disorder: Implications for serving youth who show severe aggressive and antisocial behavior. Psychology in the Schools, 41(8), 823-834. Frick, P.J. y Ellis, M.L. (1999). Callous-unemotional traits and subtypes of conduct disorder. Clinical Child and Family Psychology Review, 2, 149-168.

Frick, P.J. y Morris, A.S. (2004). Temperament and developmental pathways to conduct problems. Journal of Clinical Child and Adolescent Psychology, 33(1), 54-68. 
Frick, P.J., Bodin, S.D. y Barry, C.T. (2000). Psychopathic traits and conduct problems in community and clinic-referred samples of children: Further development of the psychopathy screening device. Psychological Assessment, 12, 382-393.

Frick, P.J., Cornell, A.H., Bodin, S.D., Dane, H.E., Barry, C.T. y Loney, B.R. (2003). Callous-unemotional traits and developmental pathways to severe conduct problems. Developmental Psychology, 39(2), 246-260.

Frick, P.J., Lilienfeld, S.O., Ellis, M.L., Loney, B.R. y Silverthorn, P. (1999). The association between anxiety and psychopathy dimensions in children. Journal of Abnormal Child Psychology, 27, 381-390.

Frick, P.J., O’Brien, B.S., Wootton, J. y McBurnett, K. (1994). Psychopathy and conduct problems in children. Joumal of Abnormal Psychology, 103, 700-707.

Frick, P.J., Stickle, T.R., Dandreaux, D., Farell, J. y Kimonis, E. (2005). Callous-unemotional traits in predicting the severity and stability of conduct problems and delincuency. Journal of Abnormal Child Psychology, 33, 471-487.

Gustafson, S.B. y Ritzer, D.R. (1995). The dark side of normal: A psychopathy-linked pattern called aberrant self-promotion. European Joumal of Personality, 9, 147-183.

Happé, F. y Frith, U. (1996). Theory of mind and social impairment in children with conduct disorder. British Journal of Development Psychology, 14, 385-398.

Hastings, P.D., Zahn-Waxler, C., Robinson, J., Usher, B. y Bridges, D. (2000). The development of concern for others in children with behavior problems. Developmental Psychology, 36(5), 531-546. Hawes, D.J. y Dadds, M.R. (2005). The treatment of conduct problems in children with callous-unemotional traits. Journal of Consulting and Clinical Psychology, 73(4), 737-741.

Hinshaw, S.P. (2002). Process, mechanism and explanation related to externalizing behavior in developmental psychopathology. Joumal of Abnormal Child Psychology, 30(5), 431-446.

Hoffman, M.L. (1975). Developmental synthesis of affect and cognition and its implication for altruistic motivation. Developmental Psychology, 11, 607-622. 
Hughes, C., Dunn, J. y White, A. (1998). Trick or treat?: Uneven understanding of mind and emotion and executive function among "hard to manage" preschoolers. Journal of Child Psychology and Psychiatry, 39(7), 981-994.

Keenan, K. y Shaw, D. (1997). Developmental and social influences on young girls's early problem behavior. Psychological Bulletin, $121,95-113$.

Kochanska, G. (1997). Multiple pathways to conscience for children with different temperaments from toddlerhood to age 5 . Developmental Psychology, 33, 228-240.

Kosson, D.S., Cyterski, T.D., Steuerwald, B.L., Newman, C.S. y WalterMatthews, S. (2002). The reliability and validity of the Psychopathy Checklist-Youth Version in non-incarcerated males. Psychological Assessment, 14, 97-109.

Lahey, B.B. y Loeber, R. (1994). Framework for a developmental model of oppositional defiant disorder and conduct disorder. En D.K. Routh (Ed), Disruptive behavior disorders in childhood (pp.139-180). New Jork: Plenum.

Lengua, L.J. (2003). Associations among emotionality, self-regulation, adjustment problems and positive adjustment in middle childhood. Applied Developmental Psychology, 24, 595-618.

Lengua, L.J. y Long, A.C. (2002). The role of emotionality and selfregulation in the appraisal-coping process: Tests of direct and moderating effects. Applied Developmental Psychology, 23, 471-493. Lima, E.N. (2004). Emotional reactivity, trait affectivity and child conduct problems. Dissertation. The Florida State University College of Sciences.

Loney, B.R., Frick, P.J., Clements, C.B., Ellis, M.L. y Kerlin, K. (2003). Callous-unemotional traits, impulsivity and emotional processing in adolescents with antisocial behavior problems. Journal of Clinical Child and Adolescent Psychology. 32(1), 66-80.

Loney, B.R., Lima, E.N. y Butler, M.A. (2006). Trait affectivity and nonreferred adolescent conduct problems. Journal of Clinical Child and Adolescent Psychology, 35(2), 329-336.

Lynam, D.R. (1998). Early identification of the psychopath: Locating the psychopathic child in the current nomenclature. Journal of Abnormal Psychology, 107, 566-575. 
Lynam, D.R., Caspi, A., Moffit, T.E., Raine, A., Loeber, R. y StouthamerLoeber, M. (2005). Adolescent psychopathy and the Big Five: Results from two samples. Journal of Abnormal Child Psychology, 33(4),431-443.

Marsee, M.A., Silverthorn, P. y Frick, P.J. (2005). The association of psychopathic traits with aggression and delinquency in non-referred boys and girls. Behavioral Sciences and the Law, 23, 1-15.

Martel, M.M. y Nigg, J.T. (2006). Child ADHD and personality/ temperament traits of reactive and effortful control, resiliency and emotionality. Journal of Child Psychology and Psychiatry (en prensa).

Masten, A.S. (2001). Ordinary magic: Resilience processes in development. American Psychologist, 56(3), 227-238.

McCord, W. y McCord, J. (1959/1964). The psychopath: An essay on the criminal mind. Princeton, $\mathrm{NJ}$ : Van Nostrand Company.

Melnick, S. y Hinshaw, S.P. (2000). Emotion regulation and parenting in $A D / H D$ and comparison boys: Linkages with social behaviors and peer preference. Journal of Abnormal Child Psychology, 28, 73-86.

Millon, T. (1997). DSM narcissistic personality disorder: Historical reflections and future directions. En E.F.Ronningstam (Ed.), Disorders of narcissism: Diagnostic, clinical and empirical implications (pp.75-101). Washington, DC:American Psychiatric Press.

Moffitt, T.E. (1993). "Life-course-persistent" and "adolescent-limited" antisocial behavior: A developmental taxonomy. Psychological Review, 100, 674-701.

Moffitt, T.E. y Caspi, A. (2001). Childhood predictors differentiate lifecourse persistent and adolescence-limited antisocial pathways among males and females. Development and Psychopathology, 13, 355-375.

Morf, C.C. y Rhodewalt, F. (2001). Unraveling the paradoxes of narcissism: A dynamic self-regulatory processing model. Psychological Inquiry, 12,177-196.

Mullin, B.C. y Hinshaw, S.P. (2006). Emotion regulation and extemalizing disorders. En J. Gross (Ed.), Handbook of emotion regulation. N.J.: Guilford. 
Newman, J.P. (1998). Psychopathic behavior: An information processing perspective. En D.J. Cooke, A.E. Forth y R.D. Hare (Eds.), Psychopathy: Theory, research and implications for society (pp.81-104). Dordrecht: Kluwer.

Odgers, C. y Moretti, M. (2002). Aggressive and antisocial girls: Research update and challenges. International Journal of Forensic Mental Health, 1, 103-119.

Olson, S.L., Sameroff, A.J., Kerr, D.C.R., López, N.L. y Wellman, H.M. (2005). Developmental foundations of externalizing problems in young children: The role of effortful control. Development and Psychopathology, 17, 25-45.

Pardini, D., Obradovic, J. y Loeber, R. (2006). Interpersonal callousness, hyperactivity / impulsivity, inattention and conduct problems as precursors to delincuency persistence in boys: A comparison of three grade-based cohorts. Joumal of Clinical Child and Adolescent Psychology, 35(1), 46-59.

Pardini, D.A., Lochman, J.E. y Frick, P.J. (2003). Callous-unemotional traits and social-cognitive processes in adjudicated youth. Joumal of the American Academy of Child and Adolescent Psychiatry, 42, 364-371.

Patrick, C.J. (1994). Emotion and psychopathy: Startling new insights. Psychophysiology, 31, 319-330.

Perner, J. y Wimmer, H. (1985). "John thinks that Mary thinks that..." Attribution of second-order beliefs by 5-10 years old children. Joumal of Experimental Child Psychology, 39, 437-471.

Perner, J., Kain, W. y Barchfeld, P. (2002). Executive control and higher-order theory of mind in children at risk of ADHD. Infant and Child Development, 11, 141-158.

Raine, A., Reynolds, C., Venables, P.H., Mednick, S.A.y Farrington, D.P. (1998). Fearlessness, stimulation-seeking and large body size at age 3 years as early predispositions to childhood aggression at age 11 years. Archives of General Psychiatry, 55, 745-751. Repacholi, B. y Slaughter V. (2003). Individual differences in theory of mind. New Jork: Psychological Press

Rhodewalt, F. y Morf, C.C. (1998). On self-aggrandizement and anger: A temporal analysis of narcissism and affective reactions to success and failure. Joumal of Personality and Social Psychology, 74, 672-685. 
Riviére, A. y Núñez, M. (1996). La mirada mental. Aique Grupo Editor. Rothbart, M.K. y Bates, J.E. (1998). Temperament. En W. Damon y

N. Eisenberg (Eds.), Handbook of child psychology (Vol 3, pp.105176). New Jork: Wiley y Sons.

Russell, J. (1996). Agency and its role in mental development. Hove: Psychology.

Salekin, R.T y Frick, P.J. (2005). Psychopathy in children and adolescents: The need for a developmental perspective. Journal of Abnormal Child Psychology, 33(4), 403-409.

Sameroff, A.J. y Mackenzie, M.J. (2003). Research strategies for capturing transactional models of development: The limits of the possible. Development and Psychopathology, 15, 613-640.

Shields, A. y Cicchetti, D. (1997). Emotion regulation among schoolage children: The development and validation of a new criterion qsort scale. Developmental Psychology, 33(6), 906-916.

Shields, A. y Cicchetti, D. (1998). Reactive aggression among maltreated children: The contributions of attention and emotion dysregulation. Journal of Clinical Child Psychology, 27(4), 381-395. Shields, A. y Cicchetti, D. (2001). Parental maltreatment and emotion dysregulation as risks factors for bullying and victimization in middle childhood. Journal of Clinical Child Psychology, 30, 349-363.

Silverthorn, P. y Frick, P.J. (1999). Developmental pathways to antisocial behavior: The delayed-onset pathway in girls. Development and Psychopathology, 11, 101-126.

Strauman, T.J. (2001). Self-regulation, affect regulation and narcissism: Pieces of the puzzle. Psychological Inquiry, 12(4), 239-242.

Sutton, J., Reeves, M. y Keogh, E. (2000). Disruptive behavior, avoidance of responsibility and theory of mind. British Journal of Developmental Psychology, 18, 1-11

Sutton, J., Smith, P.K. y Swettenham, J. (1999). Bullying and theory of mind: A critique of the social skills deficit view of antisocial behavior. Social Development, 8(1),117-127.

Thompson, R.A. (1994). Emotion regulation: A theme in search of a definition. Monographs of the Society for Research in Child Development, 59, 25-52.

Valiente, C., Eisenberg, N., Smith, C.L., Reiser, M., Fabes, R.A., Losoya, S., (2003). The relations of effortful control and reactive 
control to children's externalizing problems: A longitudinal assessment. Journal of Personality, 71, 1179-1205.

Viding, E. (2004). Annotation: Understanding the development of psychopathy. Journal of Child Psychology and Psychiatry, 45(8), 1329-1337.

Vitale, J.E., Newman, J.P., Bates, J.E., Goodnight, J., Dodge, K.A. y Pettit, G.S. (2005). Deficient behavioral inhibition and anomalous selective attention in a community sample of adolescents with psychopathic traits and low-anxiety traits. Journal of Abnormal Child Psychology, 33(4), 461-470.

Whisman, M.A. y McClelland, G.H. (2005). Designing, testing and interpreting interactions and moderator effects in family research. Journal of Family Psychology, 19(1), 111-120.

Wooton, J., Frick, P., Shelton, K. y Silverthorn, P. (1997). Ineffective parenting and childhood conduct problems: The moderating role of callous-unemotional traits. Journal of Consulting and Clinical Psychology, 65, 301-308. 


\section{FIGURAS}

Figura 1. Representación gráfica de las pendientes simples del efecto Interactivo de tres vías entre el rasgo impulsividad/irresponsabilidad, inferencia de tristeza de segundo orden y género en problemas de atención

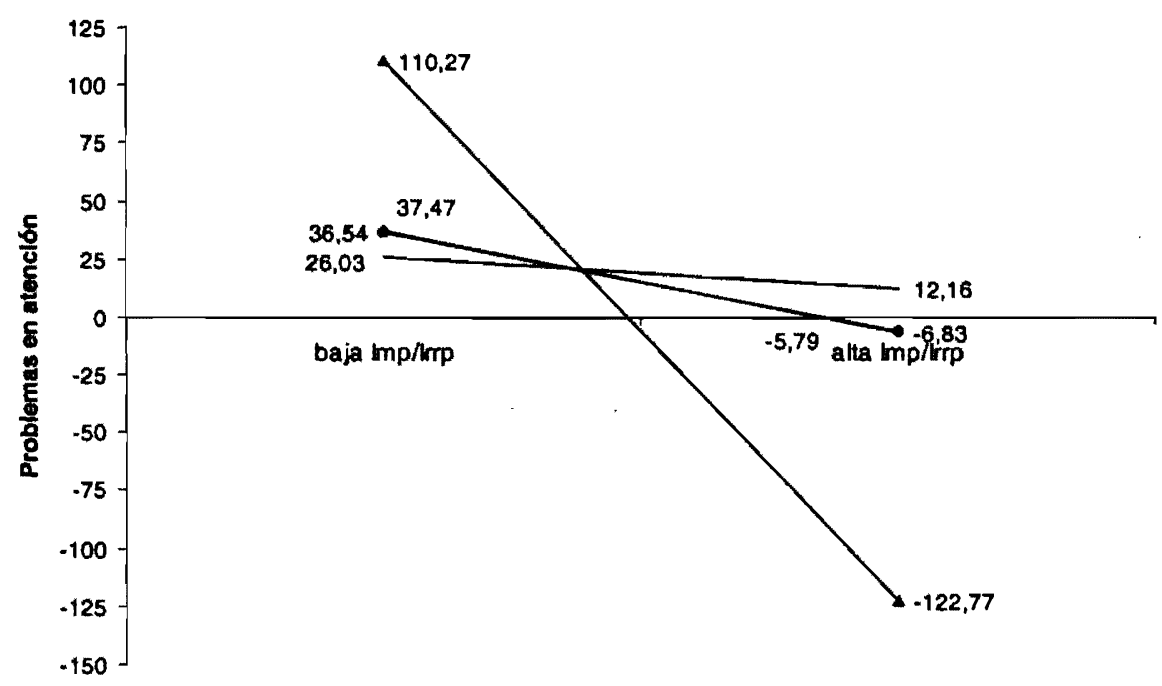

Impulsividad/Irresponsabllidad

$\begin{array}{ll}- \text { (a) alta ht.Trist género femenino" } & \rightarrow \text { (b) baja inf.Trist género femenino** } \\ \rightarrow-\text { (c) alta hf.Trist género masculino".. } & - \text { (d) baja hf.Trist género masculino (n.s.) }\end{array}$

NOTA: baja Imp/lrrp $=-14,77$, puntuación mínima obtenida por la muestra en el rasgo impulsividad / irresponsabilidad; alta $\mathrm{Imp} / \mathrm{lrp}=23,23$ correspondiente a la puntuación máxima obtenida por la muestra en el rasgo impulsividad / irresponsabilidad; InfTrist = inferencia de tristeza de segundo orden.

Significación de las pendientes simples: * $(p<.005) ;^{* \star}(p<.025){ }^{* * *}(p<.10)$ Diferencias significativas entre pendientes simples:

(a) - (b), $t=-2.402(p<.010)$

(a) - (c), $t=-2.159(p<.025)$

(a) - (d), $t=-2.453(p<.010)$

(b) - (d), $t=-1.079(p<.10)$

Las pendientes simples (b) y (c) con puntuaciones mínima y máxima $-6,83$ y $37,47, y$

$-5,79$ y 36,54 , respectivamente, no se distinguen en la representación dada su superposición en la gráfica. 


\section{Gráflco b. Género masculino}

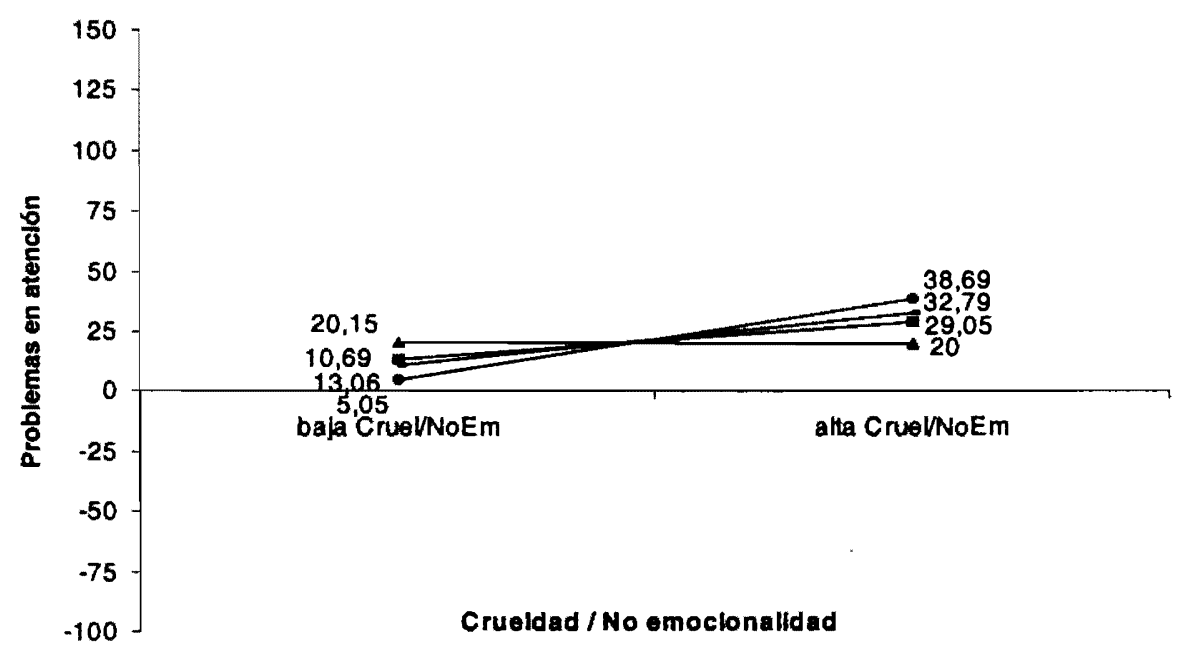

- (e) alta RegEm alta ht.Trist (n.s.) $\rightleftharpoons$ (f) baja RegEm alta Inf.Trist (n.s.)

$\rightarrow$ (g)alta RegEm baja hl.Trist ** $\rightarrow$ (h) baja RegEm baja $h t$. Trist $^{* * *}$

NOTA: Las ocho pendientes simples de la interacción de cuatro vías Crueldad / No emocionalidad, regulación emocional, inferencia de tristeza y género en problemas de atención están representadas en los dos gráficos a y b según el género.

baja Cruel/NoEm $=-14,77$, puntuación mínima obtenida por la muestra en el rasgo crueldad / no emocionalidad; alta Cruel/ $\mathrm{NoEm}=23,23$ correspondiente a la puntuación máxima obtenida por la muestra en crueldad / no emocionalidad; RegEm = regulación emocional; InfTrist = inferencia de tristeza de segundo orden.

Significación de las pendientes simples: * $(p<.005) ;{ }^{* *}(p<.050) ;{ }^{* * *}(p<.10)$ Diferencias significativas entre pendientes simples:

(b) $-(g), t=2.432(p<.010)$

(a) $-(h), t=2.497(p<.010)$

En el gráfico $b$, las pendientes simples (e) y (h) con puntuaciones mínima y máxima 10,69 y 32,79 , y 13,06 y 29,05 , respectivamente, no se distinguen en la representación dada su superposición. 